A CONTINUOUS FIELD OF PROJECTIONLESS $C^{*}$-ALGEBRAS

by

Andrew James Dean

A thesis submitted in conformity with the requirements for the degree of Doctor of Philosophy, Graduate Department of Mathematics, University of Toronto.

(C) Copyright by Andrew James Dean 1999 
National Library

of Canada

Acquisitions and Bibliographic Services

395 Wellington Street

Ottawa ON K1A ON4

Canada
Bibliothèque nationale du Canada

Acquisitions et services bibliographiques

395, rue Wellington

Ottawa ON K1A ON4

Canada
The author has granted a nonexclusive licence allowing the National Library of Canada to reproduce, loan, distribute or sell copies of this thesis in microform, paper or electronic formats.

The author retains ownership of the copyright in this thesis. Neither the thesis nor substantial extracts from it may be printed or otherwise reproduced without the author's permission.
L'auteur a accordé une licence non exclusive permettant à la Bibliothèque nationale du Canada de reproduire, prêter, distribuer ou vendre des copies de cette thèse sous la forme de microfiche/film, de reproduction sur papier ou sur format électronique.

L'auteur conserve la propriété du droit d'auteur qui protège cette thèse. $\mathrm{Ni}$ la thèse ni des extraits substantiels de celle-ci ne doivent être imprimés ou autrement reproduits sans son autorisation. 


\title{
A CONTINUOUS FIELD OF PROJECTIONLESS $C^{*}$-ALGEBRAS
}

Ph.D. Thesis 1999

Andrew James Dean

Department of Mathematics, University of Toronto.

\begin{abstract}
In this paper we shall use the fact that a certain family of crossed products of the Cuntz algebra by the reals forms a continuous field, along with a careful analysis of some of the fibres, to show that many of the other members that are difficult to analyse directly are inductive limits of pullbacks of finite direct sums of matrix algebras over the continuous functions on the unit interval by finite dimensional $C^{*}$-algebras.
\end{abstract}




\section{Acknowlegements}

The author wishes to thank his supervisor, G. A. Elliott, for his good counsel, The Fields Institute and the University of Copenhagen for their hospitality, and the Department of Mathematics and NSERC for funding. 


\section{Contents}

§1. Introduction . . . . . . . . . . . . . . . . 1

$\S 2$. The Case of Rational $\lambda$, Part I . . . . . . . . . . . . . $\quad$. 9

§3. The Case of Rational $\lambda$, Part II . . . . . . . . . . . 15

\$4. Stable Relations and the Main Result for $\mathrm{O}_{2}$. $\quad$. $\quad$. $\quad$. $\quad$. $\quad$. 28

$\S 5 . O_{n}$ and Closing Remarks . . . . . . . . . . . . 35

§6. References . . . . . . . . . . . . . . . . 40 


\section{§1. Introduction}

The subject of this thesis is a structure theorem for a class of $C^{*}$-algebras. We shall show that certain $C^{*}$-algebras built from actions of $\mathbb{R}$ on the Cuntz algebra $O_{n}$ can also be described as inductive limits of 'nice' type I $C^{*}$ algebras. This result is important to what has become known as the 'Elliott classification program.' In this introductory section we shall first review the construction of crossed product $C^{*}$-algebras, then define the particular ones we are going to study; and, finally, discuss the Elliott program and the particular result we are going to prove.

A crossed product $C^{*}$-algebra is is built from three things: a $C^{*}$-algebra $A$, a locally compact group $G$, and an action $\alpha: G \rightarrow \operatorname{Aut}(A)$ of $G$ on $A$ by *. automorphisms. The action $\alpha$ will always be assumed to be continuous with respect to the topology of norm convergence at each point. Such a triple $(A, G, \alpha)$ is called a $C^{*}$-dynamical system.

Given a $C^{*}$-dynamical system $(A, G, \alpha)$, a covariant representation of the system consists of a triple $(H, \pi, \lambda)$, where $H$ is a Hilbert space, $\pi$ is a $*_{\text {_ }}$ homomorphism mapping $A$ into $B(H)$, and $\lambda$ is a unitary representation of $G$ on $H$, such that $\pi\left(\alpha_{g}(x)\right)=\lambda_{g} \pi(x) \lambda_{g}^{*}$ for all $x \in A, g \in G$. Any $C^{*}-$ dynamical system possesses a covariant representation. If $\pi_{u}: A \rightarrow B(H)$ is the universal representation of $A$, and $\lambda: G \rightarrow L^{2}(G)$ is the left regular representation of $G$, given by $\left(\lambda_{g} \eta\right)(t)=\eta\left(g^{-1} t\right)$, then we may build a covariant

representation $\left(L^{2}(G, H), \tilde{\pi}, \tilde{\lambda}\right)$ of $(A, G, \alpha)$ as follows: $\left(\tilde{\lambda}_{g} \eta\right)(t)=\eta\left(g^{-1} t\right)$; $(\tilde{\pi}(a) \eta)(t)=\pi_{u}\left(\alpha_{t^{-1}}(a)\right) \eta(t)$. This particular covariant representation will be referred to as the regular representation of the $C^{*}$-dynamical system.

Given a $C^{*}$-dynamical system $(A, G, \alpha)$, one may make the Banach space $L^{1}(G, A)$ of $L^{1}$ functions from $G$ into $A$ into a *-algebra with the following 
operations:

$$
\begin{aligned}
f \times g(s) & =\int f(t) \alpha_{t}\left(g\left(t^{-1} s\right)\right) d t \\
f^{*}(s) & =\Delta(s)^{-1} \alpha_{s}\left(f\left(s^{-1}\right)\right)^{*} .
\end{aligned}
$$

(In the above expression $\triangle$ denotes the modular function for the group and the integrals are defined weakly.) These operations are chosen so that any covariant representation of the $C^{*}$-dynamical system, $(H, \pi, \lambda)$ say, gives rise to a representation $(\pi \times \lambda)$ of the Banach *-algebra $L^{1}(G, A)$ on $B(H)$ by the formula $(\pi \times \lambda)(f)=\int \pi(f(t)) \lambda_{t} d t$, where the integral is defined weakly, i.e. $(\pi \times \lambda)(f)$ is the unique element of $B(H)$ such that for all $\eta, \nu \in H,\langle(\pi \times \lambda)(f) \eta \mid \nu\rangle=\int\left\langle\pi(f(t)) \lambda_{t} \eta \mid \nu\right\rangle d t$. Using this map, for each covariant representation of the $C^{*}$-dynamical system $(A, G, \alpha)$ one gets a $C^{*}$ semi-norm on the Banach *-algebra $L^{1}(G, A)$, and all such semi-norms are bounded above by the $L^{1}$ norm. We may now make the following definition.

Definition 1.1 The supremum over all of the $C^{*}$-semi-norms on $L^{1}(G, A)$ coming from covariant representations of the $C^{*}$-dynamical system $(A, G, \alpha)$ is a $C^{*}$-norm. The completion of $L^{1}(G, A)$ in this norm is a $C^{*}$-algebra, called the full crossed product, or universal crossed product, of the system $(A, G, \alpha)$, and is denoted $A \rtimes_{\alpha} G$.

One may also use just the $C^{*}$-norm coming from the regular representation of $(A, G, \alpha)$, which leads to the following definition.

Definition 1.2 The completion of the *-algebra $L^{1}(G, A)$ in the $C^{*}$-norm coming from the regular representation of the $C^{*}$-dynamical system $(A, G, \alpha)$ is called the reduced crossed product of the system and is denoted $A \rtimes_{\alpha r} G$. The natural notion of isomorphism of $C^{*}$-dynamical systems is called covariant isomorphism. Two $C^{*}$-dynamical systems $(A, G, \alpha)$ and $(B, G, \beta)$ are 
said to be covariantly isomorphic if there exists a ${ }^{*}$-isomorphism $\varphi: A \rightarrow B$ such that $\varphi\left(\alpha_{g}(a)\right)=\beta_{g}(\varphi(a))$ for all $a \in A, g \in G$. Covariantly isomorphic $C^{*}$-dynamical systems give rise to isomorphic crossed products, in both the full and reduced constructions.

The identity map from $L^{1}(G, A)$ to itself extends to a surjective

*-homomorphism from $A \rtimes_{\alpha} G$ to $A \rtimes_{\alpha r} G$. In general, this map is not an isomorphism. One does however have the following theorem.

Theorem 1.3 (see [Ped] for a proof) Given a $C^{*}$-dynamical system $(A, G, \alpha)$, if the group $G$ is amenable, then the natural map from $A \rtimes_{\alpha} G$ onto $A \rtimes_{\alpha r} G$ is an isomorphism.

All abelian locally compact groups are amenable, and we shall only consider this case, so the distinction between full and reduced crossed products will no longer concern us.

Example 1.4 Let $G$ be an abelian group, and let $G$ act trivially on a $C^{*}$ algebra $A$. Then the Fourier transform $(F f)(s)=\int_{G} f(t)(t, s) d t$, where $(t, s)$ denotes the pairing of an element of $G$ with an element of $\hat{G}$, extends to an isomorphism of $A \rtimes_{i d} G$ with $C_{0}(\hat{G}, A)$. In the case of $G=\mathbb{R}$, we see that the suspension of a $C^{*}$-algebra is a special case of a crossed product.

Example 1.5 The rotation algebra $A_{\theta}$ is defined as the universal $C^{*}$-algebra generated by two unitaries $U$ and $V$ subject to the relation $U V=e^{2 \pi i \theta} V U$. This relation may be rewritten $U V U^{*}=e^{2 \pi i} V$. If one considers the crossed product $C(\mathbb{T})$ by the action $\alpha_{\theta}$ of $\mathbb{Z}$ given by rotation by $2 \pi \theta$, one sees from the definition that this is the universal $C^{*}$-algebra generated by a copy of $C(\mathbb{T})$ and a unitary $U$ such that conjugating an element of $C(\mathbb{T})$ by $U$ results in a rotation by $2 \pi \theta$. As $C(\mathbb{T})$ is the universal $C^{*}$-algebra generated by a unitary $V$, one easily concludes that $C(\mathbb{T}) \rtimes_{\alpha_{\theta}} \mathbb{Z} \cong A_{\theta}$. 
Example 1.6 Given a $C^{*}$-dynamical system $(A, G, \alpha)$ in which $G$ is an abelian group with dual group $\hat{G}$, there is an action $\hat{\alpha}$ of $\hat{G}$ on $A \rtimes_{\alpha} G$ that, on elements of $L^{1}(G, A)$, is given by $\left(\hat{\alpha}_{\sigma} f\right)(t)=(\sigma, t) f(t)$ for all $t \in G$, $\sigma \in \hat{G}$. This action $\hat{\alpha}$ is referred to as the dual action on $A \rtimes_{\alpha} G$.

In this thesis, we shall be considering crossed products coming from $C^{*}$ dynamical systems in which the $C^{*}$-algebra is one of the Cuntz algebras $O_{n}$. The algebra $O_{n}$ was defined in [Cu1] to be the universal $C^{*}$-algebra generated by $n$ isometries $S_{1}, \ldots, S_{n}$ satisfying the relation $S_{1} S_{1}^{*}+\ldots+S_{n} S_{n}^{*}=1$. Cuntz showed in [Cul] that $O_{n}$ is a simple $C^{*}$-algebra, so in fact any $n$ isometries in a $C^{*}$-algebra satisfying the relation above generate a copy of $O_{n}$. The algebra $O_{\infty}$ is defined as the universal $C^{*}$-algebra generated by a sequence $\left\{S_{n}\right\}$ of isometries such that $\sum S_{n} S_{n}^{*} \leq 1$.

There is also a Fock space construction of the $O_{n} \mathrm{~s}$, and using this Evans in [Ev] defined what he called quasi-free automorphisms of $O_{n}$. The construction is as follows. Let $F(H)$ denote the full Fock space $\bigoplus_{n=0}^{\infty}\left(\otimes^{n} H\right)$ for a separable Hilbert space $\mathrm{H}$, where $\otimes^{0} H$ denotes the one dimensional subspace spanned by the vacuum vector $\Omega$. Define a linear map $O: H \rightarrow B(F(H))$ by $O(f) \Omega=f$ and $O(f)\left(f_{1} \otimes f_{2} \otimes \ldots \otimes f_{n}\right)=f \otimes f_{1} \otimes \ldots \otimes f_{n}$. The map $O$ satisfies the relations $O(f)^{*} O(g)=\langle g \mid f\rangle 1$ and $\sum_{i=1}^{n} O\left(h_{i}\right) O\left(h_{i}\right)^{*} \leq 1$ for any orthonormal basis $\left\{h_{i}\right\}$ with equality $\bmod K(F(H))$ if $H$ is finite dimensional. Let $O(H)$ denote the $C^{*}$-algebra generated by the image of this map. If $\operatorname{dim} H=\infty$ then $O(H) \cong O_{\infty}$, and if $\operatorname{dim} H=n<\infty$ then $O(H) / K(F(H)) \cong O_{n}$. If $U$ is a unitary on $H$, then $U$ gives rise to an automorphism of $O(H)$, denoted $O(U)$, such that $O(U) O(f)=O(U f)$ for all $f \in H$. These in turn induce automorphisms of the $O_{n} \mathrm{~s}$, and such automorphisms are called quasi-free. 
In this paper, we shall consider the crossed products of $O_{n}$ by $\mathbb{R}$ actions induced by one-parameter subgroups of $U(n)$. Let $\left\{e_{1}, \ldots, e_{n}\right\}$ be an orthonormal basis for $\mathbb{C}^{n}$ and let $S_{1}=\pi \circ O\left(e_{1}\right), \ldots, S_{n}=\pi \circ O\left(e_{n}\right)$, where $\pi$ is the quotient map from $O\left(\mathbb{C}^{n}\right)$ onto $O_{n}$. Then, up to covariant isomorphism, all such automorphism groups are of the form $\alpha_{t}\left(S_{1}\right)=e^{2 \pi i \lambda_{1} t} S_{1}, \ldots, \alpha_{t}\left(S_{n}\right)=$ $e^{2 \pi i \lambda_{n} t} S_{n}$ for some real numbers $\lambda_{1}, \ldots, \lambda_{n}$. If all of the $\lambda$ s are zero, then we have just a trivial action and the crossed product is the suspension of $O_{n}$. If we assume that $\lambda_{1} \neq 0$ then, since a real crossed product is not changed, up to isomorphism, by scaling the real parameter, we may assume that $\lambda_{1}=1$. We then have an $(n-1)$-parameter family of crossed products $O_{n} \rtimes_{\alpha^{\lambda}} \mathbb{R}$, where $\lambda=\left(\lambda_{2}, \ldots, \lambda_{n}\right)$.

In [Ks] Kishimoto showed that $O_{n} \rtimes_{\alpha^{\lambda}} \mathbb{R}$ is simple if and only if one of the following two conditions holds:

1. $\left\{1, \lambda_{2}, \ldots, \lambda_{n}\right\}$ generates $\mathbb{R}$ as a closed subgroup and $\lambda_{2}, \ldots, \lambda_{n}$ are all positive.

2. $\left\{1, \lambda_{2}, \ldots, \lambda_{n}\right\}$ generates $\mathbb{R}$ as a closed subsemigroup.

For the special case of $\mathrm{O}_{2}$, this reduces to saying that $\lambda_{2}$ is irrational, case 1 holding when $\lambda_{2}>0$, case 2 holding when $\lambda_{2}<0$. In [KK2] Kishimoto and Kumjian showed that in either of the above cases $O_{n} \rtimes_{\alpha^{\lambda}} \mathbb{R}$ is stable. This places these simple crossed products of $O_{n}$ in the class of $C^{*}$-algebras targeted for classification by the Elliott program.

Elliott has conjectured that all stable, simple, separable, nuclear $C^{*}$-algebras not of type I are characterised up to isomorphism by K-theoretic and tracial invariants. More precisely, the proposed invariant consists of the $K_{1}$ group, the ordered $K_{0}$ group, the cone of densely defined, lower semi-continuous traces, denoted $T^{+}$, and the natural pairing between $K_{0}$ and $T^{+}$. A proof 
of this conjecture would generalise many known results in this direction. We give the following two examples.(see [E2] for a survey.)

Example 1.7 A $C^{*}$-algebra is called approximately finite dimensional, or $\mathrm{AF}$, if it is the closure of an increasing union of finite dimensional sub$C^{*}$-algebras. These include the uniformly hyperfinite, or UHF, algebras of Glimm, in particular the CAR algebra, also known as $M_{2 \infty}$. In [Br] Bratteli gave the first classification of these algebras, in terms of diagrams displaying the multiplicity data for the sequences of finite dimensional algebras. In [E1], Elliott showed that they could be classified in terms of the invariant described above. In [EHS], Effros, Handelman, and Shen completed the classification picture for AF algebras by characterising precisely the class of ordered abelian groups that occur as $K_{0}$ of an AF algebra (the invariant reduces to just the ordered $K_{0}$ in the case of an AF algebra, as $K_{1}$ is zero and the tracial data is redundant).

Example 1.8 A $C^{*}$-algebra is called purely infinite if every hereditary sub- $C^{*}$-algebra contains an infinite projection, in other words, one that is Murray-von Neumann equivalent to a proper subprojection of itself. It has been shown by Kirchberg and Phillips (cf. [K] and [KP]) that simple, separable, nuclear, stable, purely infinite $C^{*}$-algebras satisfying a condition on their K-theory called the universal coefficient theorem are classified up to isomorphism by their $K_{0}$ and $K_{1}$ groups (for the algebras in this class the positive cone of $K_{0}$ is the whole group and $T^{+}=0$ ). The Cuntz algebras $O_{n}$ described above are examples of $C^{*}$-algebras in this class. Also in [KK2] Kishimoto and Kumjian showed that in case 2 above $O_{n} \rtimes_{\alpha^{\lambda}} \mathbb{R}$ is in this class.

It can be shown (cf. [E3]) that the classification conjecture divides into three 
disjoint cases as follows.

$$
\begin{aligned}
\text { case I } & K_{0}^{+}=0, \quad T^{+} \neq 0 \\
\text { case II } & K_{0}^{+} \cap-K_{0}^{+}=0, \quad K_{0}^{+}-K_{0}^{+}=K_{0}, \quad T^{+} \neq 0 \\
\text { case III } & K_{0}^{+}=K_{0}, \quad T^{+}=0
\end{aligned}
$$

The AF algebras of example 1.7 fall under case II, and the purely infinite $C^{*}$ algebras of example 1.8 fall under case III, in fact, they may be all of case III. Case I consists of projectionless $C^{*}$-algebras. The first example of an algebra in this class was given by Blackadar in [BI1]. In [KK1], Kishimoto and Kumjian showed that if the first condition for simplicity is satisfied $O_{n} \rtimes_{\alpha^{\lambda}} \mathbb{R}$ is in this class.

In [Cu2], Cuntz computed the K-theory of the $O_{n}$ 's: $K_{0}\left(O_{n}\right) \cong \mathbb{Z} /(n-$ $1) \mathbb{Z}$ and $K_{1}\left(O_{n}\right) \cong 0$. In [Co], Connes showed that for any $C^{*}$-dynamical system $(A, \mathbb{R}, \alpha), K_{0}\left(A \rtimes_{\alpha} \mathbb{R}\right) \cong K_{1}(A)$ and $K_{1}\left(A \rtimes_{\alpha} \mathbb{R}\right) \cong K_{0}(A)$. In [KK1], Kishimoto and Kumjian showed that in the first of their two cases $O_{n} \rtimes_{\alpha^{\lambda}} \mathbb{R}$ has a unique trace. Thus we have that the invariants for the projectionless crossed products of $O_{n}$ given above are $K_{0} \cong 0, K_{1} \cong \mathbb{Z} /(n-1) \mathbb{Z}$, and $T^{+} \cong \mathbb{R}^{+}$.

In [E3], Elliott showed that a wide variety of values of the above invariant occur for $C^{*}$-algebras satisfying the classification hypotheses and arising as inductive limits of type I $C^{*}$-algebras. Among the values given by Elliott's construction are those of the simple projectionless crossed products of $O_{n}$ mentioned above. This suggested that these algebras could be inductive limits of type I $C^{*}$-algebras. The object of this thesis is to show that, for a dense category 2 subset of the positive multi-indices, the crossed product $O_{n} \rtimes_{\alpha^{\lambda}} \mathbb{R}$ is an inductive limit of pullbacks of finite direct sums of matrix algebras over the unit interval by finite dimensional $C^{*}$-algebras. The proofs 
for general $\mathrm{O}_{n}$ are virtually the same as for the special case of $\mathrm{O}_{2}$, with some additional bookkeeping. Thus for most of the paper we shall concentrate on $\mathrm{O}_{2}$ and leave the general case to section $\S 5$, where we also deal with some other questions raised along the way. The proof requires that we first undertake a careful analysis of $\mathrm{O}_{2} \rtimes_{\alpha^{\lambda}} \mathbb{R}$ where $\lambda$ is a rational number different from 1. This analysis is carried out in $\S 2$ and $\S 3$. The proof of the main result for the case of $\mathrm{O}_{2}$ is then given in $\S 4$. 


\section{$\S 2$. The Case of Rational $\lambda$, Part I}

In this section we shall begin to analyse the crossed products $\mathrm{O}_{2} \rtimes_{\alpha^{\lambda}} \mathbb{R}$ for $\lambda$ a rational number not equal to one. Using elementary properties of the constructions, we shall deduce descriptions for them as mapping tori over algebras whose generators satisfy relations that we can describe using diagrams.

Suppose $\lambda=p / q$ is a rational number expressed in lowest terms. Then the action $\alpha^{\lambda}$ is periodic with period $q$. We make the change of variables $t \mapsto q t$ to get an action with period 1 for which the crossed product is isomorphic. For the remainder of this section we shall call this new action $\alpha$, explicitly, $\alpha_{t}\left(S_{1}\right)=e^{2 \pi i q t} S_{1}, \alpha_{t}\left(S_{2}\right)=e^{2 \pi i p t} S_{2}$. In [B12 prop. 10.3.2] it is shown that the crossed product $\mathrm{O}_{2} \rtimes_{\alpha} \mathbb{R}$ is isomorphic to the mapping torus of $\mathrm{O}_{2} \rtimes_{\alpha} \mathbb{T}$ by an automorphism generating the dual action of $\mathbb{Z}$ (cf. [OP] for a more general version of this theorem).

To analyse these mapping tori it will be convenient to express $\mathrm{O}_{2}$ in a different way. Write $M_{2 \infty} \otimes K$ as the infinite tensor product, indexed by the integers, of copies of $M_{2}$ trailing off as the identity to the right and as $e_{11}$ to the left. (This means that $M_{2 \infty} \otimes K$ is written as the inductive limit of tensor products of finitely many copies of $M_{2}$, with the inclusions $M_{2^{n}} \hookrightarrow M_{2^{n+1}}$ given by $x \mapsto e_{11} \otimes x \otimes 1$, where we have assumed that a fixed set of matrix units is given for each $M_{2}$ factor and that the factors are indexed by the integers.) Let $\beta$ denote the automorphism of $M_{2 \infty} \otimes K$ given by shifting one position to the right in the infinite tensor product. Observe that $\beta$ scales the unique trace on $M_{2 \infty} \otimes K$ by $1 / 2$, i.e. $\tau \circ \beta=(1 / 2) \tau$. If $e$ is the unit of the 0 th copy of $M_{2}$ (i.e. $e=\ldots e_{11}^{-2} \otimes e_{11}^{-1} \otimes 1 \otimes 1 \otimes \ldots$ ), then it follows from [Rør] that $e\left(\left(M_{2 \infty} \otimes K\right) \rtimes_{\beta} \mathbb{Z}\right) e \cong O_{2}$, and in fact more is true. Define an action $\tilde{\alpha}_{t}$ of $\mathbb{T} \cong \mathbb{R} / \mathbb{Z}$ on $\left(M_{2^{\infty}} \otimes K\right) \rtimes_{\beta} \mathbb{Z}$ as follows. On $M_{2 \infty} \otimes K$ in 
its tensor product expression $\tilde{\alpha}_{t}$ is the product type automorphism given by infinitely many copies of $u_{t}=\gamma_{1}^{t} e_{11}+\gamma_{2}^{t} e_{22}$, where $\gamma_{1}^{t}$ and $\gamma_{2}^{t}$ denote $e^{2 \pi i q t}$ and $e^{2 \pi i p t}$ respectively. If $V$ is the adjoined unitary in the multiplier algebra of $\left(M_{2} \infty K\right) \rtimes_{\beta} \mathbb{Z}$ implementing $\beta$, then $\tilde{\alpha}_{t}(V)=\gamma_{1}^{t} V$. This action fixes $e$, so we get an action, also denoted $\tilde{\alpha}_{t}$, of $\mathbb{T}$ on $e\left(\left(M_{2^{\infty}} \otimes K\right) \rtimes_{\beta} \mathbb{Z}\right) e$. We then have the following.

Lemma 2.1 The $C^{*}$ dynamical systems $\left(O_{2}, \alpha_{t}, \mathbb{T}\right)$ and $\left(e\left(\left(M_{2} \infty K\right) \rtimes_{\beta}\right.\right.$ $\left.\mathbb{Z}) e, \tilde{\alpha}_{t}, \mathbb{T}\right)$ are covariantly isomorphic.

Proof: At this point we introduce a simplification in our notation. When we write $e_{i j}^{k} \otimes \ldots \otimes e_{s t}^{k+n}$ for an element of $M_{2 \infty} \otimes K$ it is assumed that all entries to the right of those shown are 1's and all those to the left are $e_{11}$ 's, so for example $e_{11}^{-1} \otimes 1^{0} \otimes 1^{1}$ is the element $e$. Let $T_{1}=V e$ and $T_{2}=\left(e_{11}^{-1} \otimes e_{21}^{0} \otimes 1^{1}\right) V e$. Then straightforward computations show that $T_{1}^{*} T_{1}=T_{2}^{*} T_{2}=e, T_{1} T_{1}^{*}+T_{2} T_{2}^{*}=e, \tilde{\alpha}_{t}\left(T_{1}\right)=\gamma_{1}^{t} T_{1}$ and $\tilde{\alpha}_{t}\left(T_{2}\right)=\gamma_{2}^{t} T_{2}$. Thus by the universal property of $\mathrm{O}_{2}$ there is an isomorphism of $\mathrm{O}_{2}$ onto the sub- $C^{*}$-algebra of $\left(M_{2 \infty} \otimes K\right) \rtimes_{\beta} \mathbb{Z}$ generated by $T_{1}$ and $T_{2}$ intertwining the actions. It remains to show that $T_{1}$ and $T_{2}$ generate all of $e\left(\left(M_{2 \infty} \otimes\right.\right.$ $\left.K) \rtimes_{\beta} \mathbb{Z}\right) e$. Notice that as $\beta^{-k}(e) \geq e$ in $\left(M_{2 \infty} \otimes K\right) \rtimes_{\beta} \mathbb{Z}$ for $k \geq 0$, we have $e A V^{k} e=(e A e)(e V e)^{k}$ for all $A \in M_{2} \otimes K$. As $e V e=T_{1}$ we have only to see that $e\left(M_{2^{\infty}} \otimes K\right) e \subseteq C^{*}\left(T_{1}, T_{2}\right)$. This follows from the observations that $e\left(M_{2 \infty} \otimes K\right) e$ is generated by those tensors with $e_{11}$ in all positions to the left of zero, that if $e A e \in C^{*}\left(T_{1}, T_{2}\right)$ for some $A \in M_{2 \infty} \otimes K$ then $\beta^{k}(e A e)=(V e)^{k} A\left(e V^{*}\right)^{k}=T_{1}^{k} A T_{1}^{* k} \in C^{*}\left(T_{1}, T_{2}\right)$ for all $k \geq 0$, and the calculations $T_{1} T_{2}^{*}=e_{21}^{0}, T_{1} T_{1}^{*}=e_{11}^{0}$ and $T_{2} T_{2}^{*}=e_{22}^{0}$, which show that the matrix units for the 0th copy of $M_{2}$ are included.

The next step in our analysis is to interchange the order of the two crossed 
products. On the dense subalgebra $C_{c}\left(\mathbb{Z}, M_{2 \infty} \otimes K\right)$ of $\left(M_{2^{\infty}} \otimes K\right) \rtimes_{\beta} \mathbb{Z}$ we have $\left(\tilde{\alpha}_{t} g\right)(k)=(t, k)^{q} \alpha_{t}(g(k))$ for all $g \in C_{c}\left(\mathbb{Z}, M_{2} \otimes K\right), t \in \mathbb{R} / \mathbb{Z}$, and $k \in \mathbb{Z}$, where $(t, k)$ denotes the pairing of an element of $\mathbb{T}$ with an element of $\mathbb{Z} \cong \hat{\mathbb{T}}$. Consider the action, denoted $\tilde{\beta}$, on $\left(M_{2 \infty} \otimes K\right) \rtimes_{\bar{\alpha}} \mathbb{T}$ defined by $\left(\tilde{\beta}_{k} f\right)(t)=\overline{(t, k)^{q}} \beta_{k}(f(t))$ for all $f \in C_{c}\left(\mathbb{T}, M_{2 \infty} \otimes K\right), t \in \mathbb{R} / \mathbb{Z}$, and $k \in \mathbb{Z}$. We then have the following lemma, which is easy to check.

Lemma 2.2 The map $\Phi: C\left(\mathbb{T}, C_{c}\left(\mathbb{Z}, M_{2 \infty} \otimes K\right)\right) \rightarrow C_{c}\left(\mathbb{Z}, C\left(\mathbb{T}, M_{2 \infty} \otimes K\right)\right)$ given by $(\Phi f)(k, t)=\overline{(t, k)}^{q} f(t, k)$ for all $t \in \mathbb{R} / \mathbb{Z}$ and $k \in \mathbb{Z}$ extends to an isomorphism of $\left(\left(M_{2 \infty} \otimes K\right) \rtimes_{\beta} \mathbb{Z}\right) \rtimes_{\bar{\alpha}} \mathbb{T}$ with $\left(\left(M_{2 \infty} \otimes K\right) \rtimes_{\bar{\alpha}} \mathbb{T}\right) \rtimes_{\tilde{\beta}} \mathbb{Z}$.

Now we dissect the crossed product $\left(M_{2^{\infty}} \otimes K\right) \rtimes_{\bar{\alpha}} \mathbb{T}$ while keeping track of $\beta$. If we view $M_{2 \infty} \otimes K$ as the limit of the inductive system $M_{2} \rightarrow$ $M_{2} \otimes M_{2} \otimes M_{2} \rightarrow \otimes^{5} M_{2} \rightarrow \ldots$, where the maps are the inclusions given by $x \mapsto e_{11} \otimes x \otimes 1$, we see that the action $\tilde{\alpha}$ is of inductive limit type; that is, it leaves invariant each of the sub- $C^{*}$-algebras in this increasing sequence. The crossed product of the limit, $M_{2} \otimes K$, is then the inductive limit of the crossed products $\left(M_{2^{2 n+1}}\right) \rtimes_{\bar{\alpha}} \mathbb{T}$ with the obvious inclusions. It is easy to check that the map $\psi_{n}$ given by $\left(\psi_{n} x\right)(t)=x(t)\left(\otimes^{2 n+1} u_{t}\right)$ for all $x \in C\left(\mathbb{T}, M_{2^{2 n+1}}\right)$ gives an isomorphism $\psi_{n}:\left(M_{2^{2 n+1}}\right) \rtimes_{\bar{\alpha}} \mathbb{T} \rightarrow\left(M_{2^{2 n+1}}\right) \rtimes_{i d} \mathbb{T}$. The Fourier transform defines an isomorphism $F:\left(M_{2^{2 n+1}}\right) \rtimes_{i d} \mathbb{T} \rightarrow\left(M_{2^{2 n+1}}\right) \otimes C_{0}(\mathbb{Z})$ by $(F f)(k)=\int_{\mathbb{R} / \mathbb{Z}} f(t)(t, k) d t$ for all $k \in \mathbb{Z}$, so we have that $\left(M_{2^{\infty}} \otimes K\right) \rtimes_{\bar{\alpha}} \mathbb{T}$ is AF. To determine its structure completely we have only to check how the inclusions of the $\left(M_{2^{2 n+1}}\right) \rtimes_{\bar{\alpha}}$ T's one into the next are transformed by the map $\psi_{n}$ and the Fourier transform. We get a diagram:

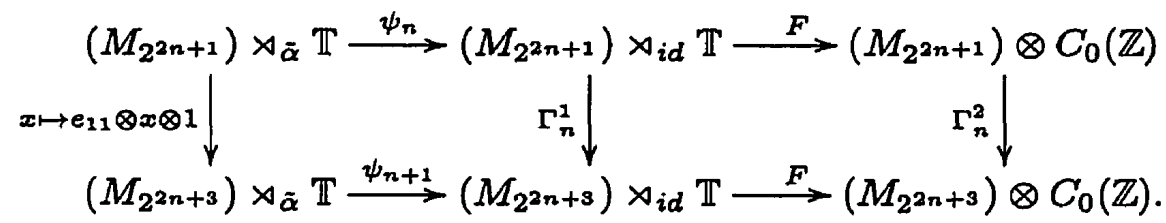


For the first square we see that $\left(\Gamma_{n}^{1} y\right)(t)=\gamma_{1}^{t}\left(e_{11} \otimes y(t) \otimes u_{t}\right)$ for all $t \in \mathbb{T}$, $y \in C\left(\mathbb{T}, M_{2^{2 n+1}}\right)$, and for the second $\left(\Gamma_{n}^{2} z\right)(k)=e_{11} \otimes\left(z(2 q+k) \otimes e_{11}+\right.$ $\left.z(q+p+k) \otimes e_{22}\right)$, for all $k \in \mathbb{Z}, z \in C_{c}\left(\mathbb{Z}, M_{2^{2 n+1}}\right)$. We thus arrive at the following description:

Lemma 2.3 $\left(M_{2 \infty} \otimes K\right) \rtimes_{\bar{\alpha}} \mathbb{T}$ is isomorphic to the limit of the inductive system $\left\{M_{2^{2 n+1}} \otimes C_{0}(\mathbb{Z}), \Gamma_{n}^{2}\right\}_{n=0}^{\infty}$.

Next we see what becomes of the automorphism $\tilde{\beta}$. We have a similar diagram:

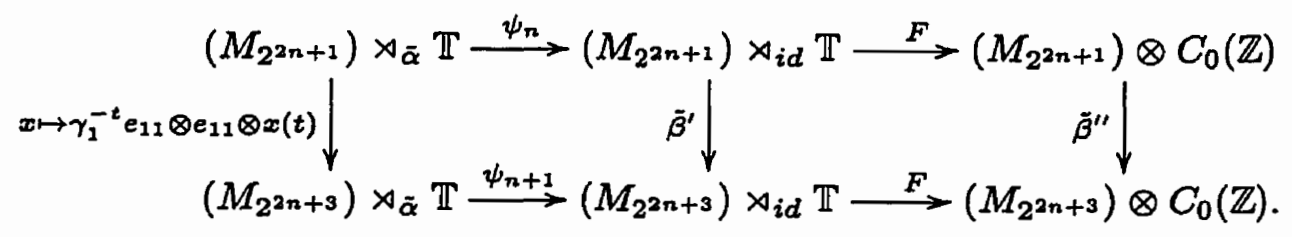

For the first square we get $\left(\tilde{\beta}^{\prime} y\right)(t)=\gamma_{1}^{t} e_{11} \otimes e_{11} \otimes y(t)$, for all $t \in \mathbb{T}$ and $y \in C\left(\mathbb{T}, M_{2^{2 n+1}}\right)$, and for the second $\left(\tilde{\beta}^{\prime \prime}(z)\right)(k)=e_{11} \otimes e_{11} \otimes z(k+q)$ for all $z \in C_{c}\left(\mathbb{Z}, M_{2^{2 n+1}}\right)$.

The dual action $\hat{\alpha}$ of $\mathbb{Z}$ on $e\left(\left(M_{2 \infty} \otimes K\right) \rtimes_{\beta} \mathbb{Z}\right) e \rtimes_{\bar{\alpha}} \mathbb{T}$ is just the restriction of the dual action of $\mathbb{Z}$, also denoted $\hat{\alpha}$, on $\left(\left(M_{2 \infty} \otimes K\right) \rtimes_{\beta} \mathbb{Z}\right) \rtimes_{\bar{\alpha}} \mathbb{T} \cong$ $\left(\left(M_{2 \infty} \otimes K\right) \rtimes_{\bar{\alpha}} \mathbb{T}\right) \rtimes_{\bar{\beta}} \mathbb{Z}$. Since $\hat{\alpha}$ commutes with $\tilde{\beta}$ to describe it we need only see what it does on $\left(M_{2 \infty} \otimes K\right) \rtimes_{\bar{\alpha}} \mathbb{T}$. We again get a diagram:

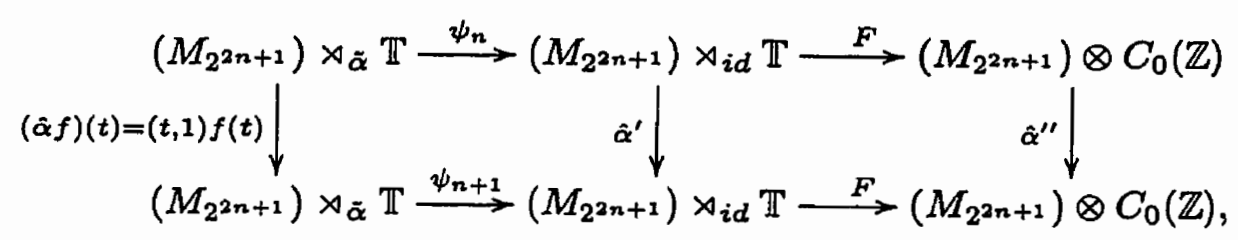

and it is easy to check that $\left(\hat{\alpha}^{\prime} f\right)(t)=(t, 1) f(t)$ for all $t \in \mathbb{T}$ and $f \in$ $C\left(\mathbb{T}, M_{2^{2 n+1}}\right),\left(\hat{\alpha}^{\prime \prime} f\right)(k)=f(k+1)$ for all $k \in \mathbb{Z}$ and $f \in C\left(\mathbb{T}, M_{2^{2 n+1}}\right)$. 
Finally, we cut down by the projection e. Cutting down the inductive system by $e$ at each stage gives us $M_{2} \rightarrow M_{2^{2}} \rightarrow M_{2^{3}} \rightarrow \ldots$, where the inclusions are $x \mapsto x \otimes 1$ at each stage. Using the same symbols for the cutdown versions of the maps found above we get:

$$
\begin{aligned}
& \tilde{\beta}^{\prime \prime}(z)(k)=e_{11} \otimes z(k+q) \\
& \left(\hat{\alpha}^{\prime \prime} f\right)(k)=f(k+1) \\
& \left(\Gamma_{n}^{2} z\right)(k)=z(2 q+k) \otimes e_{11}+z(p+q+k) \otimes e_{22},
\end{aligned}
$$

and $e\left(\left(M_{2^{\infty}} \otimes K\right) \rtimes_{\bar{\alpha}} \mathbb{T}\right) e \cong \lim _{\longrightarrow}\left\{M_{2^{n}} \otimes C_{0}(\mathbb{Z}), \Gamma_{n}^{2}\right\}$.

We may summarise this situation in the following two diagrams:

Infinite case $(\lambda=p / q<0)$

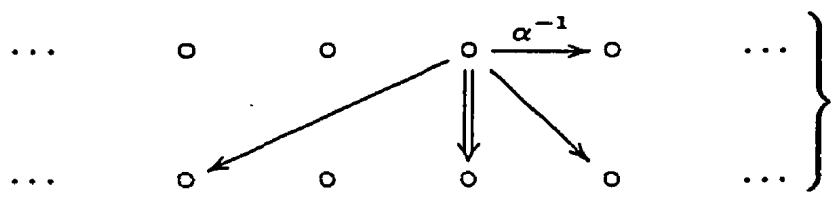

diagram 1

Finite case $(\lambda=p / q>0)$

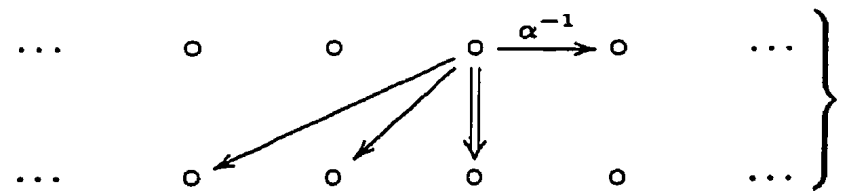

diagram 2

In these diagrams the dots and downward thin arrows represent the Bratteli diagram of the inductive system we have found for $e\left(\left(M_{2 \infty} \otimes K\right) \rtimes_{\bar{\alpha}} \mathbb{T}\right) e$. (The arrows shown are repeated at every dot in the diagram, which extends infinitely to either side and downward.) The thick downward arrow represents an adjoined partial isometry implementing $\tilde{\beta}^{\prime \prime}$ on that summand, i.e. setting a minimal projection in the upper dot equivalent to a minimal projection in the lower dot, and the arrow labelled $\alpha^{-1}$ is supposed to indicate that the automorphism $\hat{\alpha}^{\prime \prime}$ moves everything one step over. It is also to be understood that there is a compatibility between the fat arrows and the Bratteli 
diagram, the partial isometries with the same range and base spaces being identified. (All this will be precisely stated in the next section.) From this point on we shall only consider the finite case, leaving the infinite case to the closing remarks in section $\S 5$. In the next section we shall analyse universal $C^{*}$-algebras given by generators and relations corresponding to diagrams such as diagram 2 above, and we shall see that these diagrams actually give complete descriptions of the $\mathrm{O}_{2} \rtimes_{\alpha(p / q)} \mathbb{R}$ 's. 


\section{$\S 3$. The Case of Rational $\lambda$, Part II}

In this section we shall introduce universal $C^{*}$-algebras, called $A(p, q)$, which we describe in terms of generators and relations. These are designed to be universal algebras for the diagrams introduced in the previous section. We shall deduce rules for reading the structure of the $A(p, q)$ s off from these diagrams, in particular, showing that they are AF. We shall then be able to complete the analysis of the $\mathrm{O}_{2} \rtimes_{\alpha(p / q)} \mathbb{R s}$ begun in the previous section.

Definition 3.1 Let $W^{n}$ denote the set of words of length $n$ in the letters $a$, $b$. Let $p$ and $q$ be two distinct positive integers with $(p, q)=1$. Define $A(p, q)$ to be the universal $C^{*}$-algebra given by the following presentation:

\section{Generators:}

$$
\begin{aligned}
& \begin{array}{ll}
E_{w, v}^{k, n} & \text { for } k \in \mathbb{Z}, n \in \mathbb{N}, w, v \in W^{n} \\
V^{k, n} & \text { for } k \in \mathbb{Z}, n \in \mathbb{N}
\end{array} \\
& \text { Relations: } \\
& E_{w, v}^{k, n} E_{s, t}^{l, n}=0 \quad \text { if } l \neq k \\
& E_{w, v}^{k, n} E_{s, t}^{k, n}=\delta_{v s} E_{w, t}^{k, n} \\
& E_{w, v}^{k, n *}=E_{v, w}^{k, n} \\
& E_{w, v}^{k, n}=E_{w b, v b}^{k-p, n+1}+E_{w a, v a}^{k-q, n+1} \\
& V^{k, n *} V^{k, n}=\sum_{w \in W^{n}} E_{w, w}^{k, n} \\
& V^{k, n} E_{w, v}^{k, n} V^{k, n *}=E_{a w, a v}^{k, n+1} \\
& V^{k, n} E_{w, w}^{k, n}=V^{k-p, n+1} E_{w b, w b}^{k-p, n+1}+V^{k-q, n+1} E_{w a, w a}^{k-q, n+1}
\end{aligned}
$$

(In this presentation the E's correspond to the matrix units of the dots in the diagrams of the last section, and the $V$ 's are the adjoined partial isometries.) The main result of this section is the following.

Theorem 3.2 $A(p, q)$ is a simple AF algebra. Furthermore, the map $\alpha$ given below on the generators defines an automorphism, to be denoted $\hat{\alpha}$, of 
$A(p, q)$, and for $\lambda=p / q$ we have $O_{2} \rtimes_{\alpha^{\lambda}} \mathbb{R} \cong M_{\hat{\alpha}}(A(p, q))$, the mapping torus of $A(p, q)$ by $\hat{\alpha}$.

\section{Automorphism:}

$$
\alpha\left(E_{w, v}^{k, n}\right)=E_{w, v}^{k-1, n} \quad \alpha\left(V^{k, n}\right)=V^{k-1, n}
$$

We shall undertake some preliminary analysis before proving this theorem. For the sake of simplicity we shall in the following assume $q>p$, though obviously analogous statements hold with similar proofs if $p>q$. The Ktheory of $A(p, q)$ will be discussed in $\S 5$.

Let $S(n, L, K)$ denote the set of generators $\left\{E_{w, v}^{k, n} \mid w, v \in W^{n}, L-K-q \leq\right.$ $k \leq L+K\} \cup\left\{V^{k, n}|| L-k \mid \leq K\right\}$, where $L$ is any integer and $K$ is any positive integer. Let $A(S(n, L, K))$ denote the sub-C*-algebra of $A(p, q)$ generated by $S(n, L, K)$. From relations 2,3 and 5 it follows that each $V^{n, k}$ is a sum of terms $\left(V^{n, k} E_{w, w}^{k, n}\right)$. Now repeated applications of relations 4 and 7 show that for any finite set of generators $F$, the sub- $C^{*}$-algebra of $A(p, q)$ generated by the elements of $F$ is contained in some $A(S(n, L, K))$. This shows that to see that $A(p, q)$ is AF we need only look at pieces of the diagram that look like diagram 3

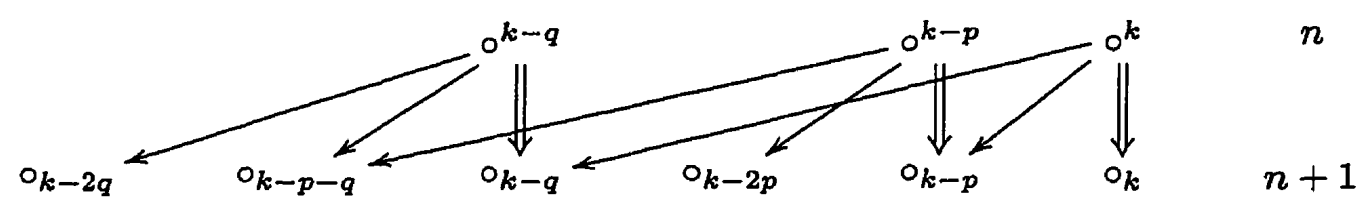

i.e. two row diagrams whose lower rows contain the ends of the arrows emanating from the dots in their upper rows. (In this diagram we have assumed $p / q<1 / 2$.) 
Let $C^{*}(S(n, L, K))$ denote the universal $C^{*}$-algebra given by the following presentation. The generators of $C^{*}(S(n, L, K))$ are identified with the elements of $S(n, L, K)$ and the relations are those in the presentation in definition 3.1 that only involve elements of $S(n, L, K)$. Clearly the identification of the generators gives a surjective map from $C^{*}(S(n, L, K))$ to $A(S(n, L, K))$.

In the following we shall refer to a diagram of the form

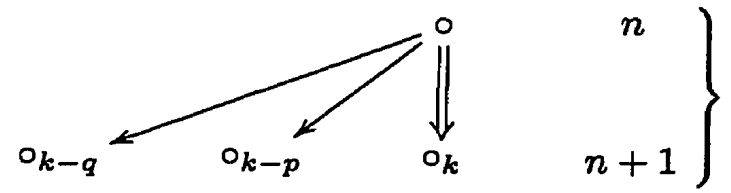

diagram 4

as a mer. The $C^{*}$-algebra generated by the elements shown in this diagram is $M_{2^{n+2}} \oplus M_{2^{n+2}}$, where $\operatorname{dot} k-q$ includes into the first summand with multiplicity one, dot $k-p$ goes into the second summand with multiplicity one, and $\operatorname{dot} k$ includes into each summand with multiplicity one. We shall use these mers to define a relation between the dots in the lower row of a two row diagram such as diagram 3 above. We shall say that dot $A$ is related by a mer to dot $B$ if there is a sub-diagram of the form of diagram 4 with $A$ as the target of the fat arrow and B as the target of a thin arrow. We shall also say that $A$ is related to $B$ by mers if there is a sequence of dots starting with $A$ and ending with $B$ with each dot related to the next (what could also be called being related by a polymer). Finally, we say that any dot is related (by mers) to itself.

Lemma 3.3 Consider the two row diagram corresponding to some set of generators $S(n, L, K)$, and consider a dot, \#(l,n+1) in the lower row that is not related by mers in this diagram to any dot lying to the left of it. Then the elements of the set $S$ described below form the first row of matrix units for a full matrix algebra contained in $C^{*}(S(n, L, K))$. Furthermore, this matrix 
algebra is a direct summand of $C^{*}(S(n, L, K))$.

Let $w \in W^{n+1}$ be distinguished as the first word in $W^{n+1}$, and fix an element $s \in W^{n}$. Then $S$ is the union of those $A_{v} s$ described below, where $v$ is a word in the letters $p$ and $q$, for which the elements of $A_{v}$ are words in the elements of $S(n, L, K)$; explicitly, moving the sum of the letters of $v$ to the right of dot \#(l,n+1) brings one to a dot still in the diagram for $S(n, L, K)$, and if $v$ begins with a $p$, then $A_{v}$ is included only if $V^{l+p, n *} \in S(n, L, K)$.

$$
\begin{aligned}
A_{0} & =\left\{E_{w, v}^{l, n+1} \mid v \in W^{n+1}\right\} \\
A_{p} & =\left\{E_{w, s b}^{l, n+1} V^{l+p, n *} E_{a s, v}^{l+p+n+1} \mid v \in W^{n+1}\right\} \\
A_{q} & =\left\{E_{w, s a}^{l, n+1} V^{l+q, n *} E_{a s, v}^{l+q, n+1} \mid v \in W^{n+1}\right\} \\
A_{p q} & =\left\{E_{w, s b}^{l, n+1} V^{l+p, n *} E_{a s, s a}^{l+p, n+1} V^{l+p+q, n *} E_{a s, v}^{l+p+q, n+1} \mid v \in W^{n+1}\right\} \\
A_{q p} & =\left\{E_{w, s a}^{l, n+1} V^{l+q, n *} E_{a s, s b}^{l+q, n+1} V^{l+p+q, n *} E_{a s, v}^{l+p+q, n+1} \mid v \in W^{n+1}\right\}
\end{aligned}
$$

and in general, if $m$ is a word in $p$ 's and $q$ 's, the sum of whose letters is $|m|$, then an element in $A_{m q}$ consists of the word in $A_{m}$ ending in $E_{a s, s a}^{l+|m|, n+1}$ followed by $V^{l+|m|+q, n *} E_{a s, v}^{l+|m|+q, n+1}$ for some $v \in W^{n+1}$, and an element of $A_{m p}$ consists of the word in $A_{m}$ ending in $E_{a s, s b}^{l+|m|, n+1}$ followed by $V^{l+|m|+p, n *} E_{a s, v}^{l+|m|+p, n+1}$ for some $v \in W^{n+1}$.

Proof: It is straightforward to check that for $v, u \in S, v^{*} v$ is a projection, $v v^{*}=u u^{*}$, and $u v^{*}=0$ if $u \neq v$, so the elements of $S$ do form the first row for a system of matrix units for a full matrix algebra. Call this matrix algebra $F_{l}$ and let $1_{F_{l}}$ denote its unit. (Strictly speaking we still have to show that $F_{l}$ is not zero, but this will be obvious from the sequel.)

To show that $F_{l}$ is a direct summand of $C^{*}(S(n, L, K))$ it will suffice to show that $1_{F_{l}}$ is in the centre of $C^{*}(S(n, L, K))$ and that $1_{F_{l}} C^{*}(S(n, L, K)) 1_{F_{l}}=$ $F_{l}$. 
To see that $1_{F_{l}}$ is in the centre of $C^{*}(S(n, L, K))$ it suffices to check that it commutes with the elements of $S(n, L, K)$. We check this first for the $E$ s. $1_{F_{l}}=\sum_{m \mid A_{m} \subseteq S}\left(\sum_{v \in A_{m}} v^{*} v\right)$. Consider an element $E_{f, g}^{k, n+1}$. As any $v \in A_{m}$ ends in a matrix unit of dot $\#(l+|m|, n+1)$, we have that $E_{f, g}^{k, n+1} \perp v^{*} v$ if $v \in$ $A_{m}$ and $l+|m| \neq k$. Suppose $l+|m|=k$ and consider $\sum_{v \in A_{m}} v^{*} v$. If $|m|=0$ then this is the unit of the $(k, n+1)$-th dot and it commutes with $E_{f, g}^{k, n+1}$. If $|m| \neq 0$ then $\sum_{v \in A_{m}} v^{*} v=\sum_{v \in A_{m}}\left(\sum_{t \in W^{n+1}} E_{t, a s}^{k, n+1} v^{*} v E_{a s, t}^{k, n+1}\right)$, and we get $E_{f, g}^{k, n+1}\left(\sum_{v \in A_{m}} v^{*} v\right)=\sum_{v \in A_{m}}\left(E_{f, a s}^{k, n+1} v^{*} v E_{a s, g}^{k, n+1}\right)=\left(\sum_{v \in A_{m}} v^{*} v\right) E_{f, g}^{k, n+1}$ Thus, we have that $1_{F_{l}}$ commutes with $E_{f, g}^{k, n+1}$.

Checking that $1_{F_{l}}$ commutes with the $V \mathrm{~s}$ in $S(n, L, K)$ is a bit more tedious. We first introduce some notation. Fix a $V^{n, k}$ in $S(n, L, K)$. Define

$$
\begin{aligned}
\Lambda & =\left\{m \mid A_{m} \subseteq S \text { and }|m|=k-l\right\} \\
\Lambda_{p} & =\left\{m \mid A_{m} \subseteq S \text { and }|m|=k-l-p\right\} \\
\Lambda_{q} & =\left\{m \mid A_{m} \subseteq S \text { and }|m|=k-l-q\right\} .
\end{aligned}
$$

Then we have that

$$
1_{F_{l}} V^{k, n}=\sum_{m \in \Lambda}\left(\sum_{v \in A_{m}} v^{*} v\right) V^{k, n}
$$

and

$$
V^{k, n} 1_{F_{l}}=\overbrace{\sum_{d \in \Lambda_{p}} V^{k, n}\left(\sum_{v \in A_{d}} v^{*} v\right)}^{\text {blob } 1}+\overbrace{\sum_{t \in \Lambda_{q}} V^{k, n}\left(\sum_{v \in A_{t}} v^{*} v\right)}^{\text {blob 2 }}
$$

where we use the convention that a sum over an empty index set gives 0 . Notice that $\Lambda=\left\{f p \mid f \in \Lambda_{p}\right\} \cup\left\{g q \mid g \in \Lambda_{q}\right\}$. (This uses that $k \neq l$. That $k=l$ is not a possibility follows from our definition of $S(n, L, K)$ and the assumption that $\operatorname{dot}(l, n+1)$ is not related by mers to any dots further left in the diagram for $S(n, L, K)$.) We then have 


$$
\sum_{m \in \Lambda}\left(\sum_{v \in A_{m}} v^{*} v\right) V^{k, n}=\overbrace{\sum_{d \in \Lambda_{p}}\left(\sum_{v \in A_{d p}} v^{*} v\right) V^{k, n}}^{\text {blob 3 }}+\overbrace{\sum_{t \in \Lambda_{q}}\left(\sum_{v \in A_{t q}} v^{*} v\right) V^{k, n}}^{\text {blob 4 }}
$$

We shall show that blob 1 is equal to blob 3 (a similar argument shows that blob 2 is equal to blob 4). Look first at blob 3.

$$
\sum_{d \in \Lambda_{p}}\left(\sum_{v \in A_{d p}} v^{*} v\right) V^{k, n}=\sum_{d \in \Lambda_{p}}\left\{\sum_{t \in W^{n+1}} E_{t, a s}^{k, n+1} V^{k, n} w_{d}^{*} w_{d} V^{k, n *} E_{a s, t}^{k, n+1} V^{k, n}\right\}
$$

where $w_{d}$ is the word in $A_{d}$ that ends in $E_{a s, s b}^{k-p, n+1}$. In the above $E_{t, a s}^{k, n+1} V^{k, n} w_{d}^{*} w_{d} V^{k, n *} E_{a s, t}^{k, n+1} V^{k, n}$ is equal to 0 if $t$ starts with a $b$, and if $t=a r$ for some $r$ in $W^{n}$ it is equal to $E_{a r, a s}^{k, n+1} V^{k, n} w_{d}^{*} w_{d} E_{s, r}^{k, n}=E_{a r, a s}^{k, n+1} V^{k, n} w_{d}^{*} w_{d} E_{s b, r b}^{k-p, n+1}$ by relations 4,5 and 6.

Thus we get:

$$
\begin{aligned}
\sum_{d \in \Lambda_{p}}\left(\sum_{v \in A_{d} p} v^{*} v\right) V^{k, n} & =\sum_{d \in \Lambda_{p}}\left\{\sum_{r \in W^{n}} E_{a r, a s}^{k, n+1} V^{k, n} w_{d}^{*} w_{d} E_{s b, r b}^{k-p, n+1}\right\} \\
& =\sum_{d \in \Lambda_{p}}\left\{\sum_{r \in W^{n}} V^{k, n} E_{r b, s b}^{k-p, n+1} w_{d}^{*} w_{d} E_{s b, r b}^{k-p, n+1}\right\}
\end{aligned}
$$

where we have used for the second equality that $E_{a r, a s}^{k, n+1} V^{k, n}=V^{k, n} E_{r, s}^{k, n}=$ $V^{k, n}\left(E_{r b, s b}^{k-p, n+1}+E_{r a, s a}^{k-q, n+1}\right)$ and that $E_{r a, s a}^{k-q, n+1} w_{d}^{*}=0$. Now we look at blob 1.

$$
\begin{aligned}
\sum_{d \in \Lambda_{p}} V^{k, n}\left(\sum_{v \in A_{d}} v^{*} v\right) & =\sum_{d \in \Lambda_{p}} V^{k, n}\left(\sum_{u \in W^{n}} E_{u b, u b}^{k-p, n+1}+E_{u a, u a}^{k-q, n+1}\right)\left(\sum_{v \in A_{d}} v^{*} v\right) \\
& =\sum_{d \in \Lambda_{p}} V^{k, n}\left(\sum_{u \in W^{n}} E_{u b, u b}^{k-p, n+1}\right)\left(\sum_{v \in A_{d}} v^{*} v\right) \\
& =\sum_{d \in \Lambda_{p}} V^{k, n}\left\{\sum_{u \in W^{n}} E_{u b, s b}^{k-p, n+1} w_{d}^{*} w_{d} E_{s b, u b}^{k-p, n+1}\right\}
\end{aligned}
$$


where the first equality follows from relations $2,3,5$ and 6 , and the second comes from the fact that elements of $A_{d}$ for $d \in \Lambda_{p}$ end in a matrix unit for $\operatorname{dot} \#(k-p, n+1)$. The last expression in the above is clearly equal to the expression for blob 3 given in equation $(\dagger)$ above. Thus we have that $1_{F_{l}}$ is in the centre of $C^{*}(S(n, L, K))$.

To show that $1_{F_{l}} C^{*}(S(n, L, K)) 1_{F_{l}}=F_{l}$ it will suffice to show that $1_{F_{l}} x 1_{F_{l}} \in$ $F_{l}$ for each element $x \in S(n, L, K)$. For any $E_{f, g}^{k, n+1}$ and $Y \in S$ either $Y E_{f, g}^{k, n+1}$ is zero or it is also in $S$. Thus $1_{F_{l}} E_{f, g}^{k, n+1} 1_{F_{l}}=\left(\sum_{Y \in S} Y^{*} Y\right) E_{f, g}^{k, n+1}$ $\left(\sum_{Y \in S} Y^{*} Y\right) \in C^{*}(S)=F_{l}$. Suppose $Y \in A_{m}$, so $Y$ ends in a matrix unit of $\operatorname{dot} \#(l+|m|, n+1)$, and suppose $V^{k, n} \in S(n, L, K)$. If $l+|m| \neq k$ then $Y V^{k, n}=0$. If $l+|m|=k$, then $|m| \neq 0$ as we have assumed that $\operatorname{dot} \#(l, n+1)$ is not related by mers to any dots further left in the diagram for $S(n, L, K)$. We have $Y=Z V^{k, n *} E_{a s, v}^{k, n+1}$ for some $v \in W^{n+1}$ and some element $Z$ of $S$ ending in either a matrix unit for $\operatorname{dot} \#(k-p, n+1)$ or one for $\operatorname{dot} \#(k-q, n+1) . Y V^{k, n}=Z\left(V^{k, n *} E_{a s, v}^{k, n+1} V^{k, n}\right)$. If $v$ begins with a $b$, then $V^{k, n *} E_{a s, v}^{k, n+1} V^{k, n}=0$, and if $v=a r$ for some $r \in W^{n}$ then $V^{k, n *} E_{a s, a r}^{k, n+1} V^{k, n}=E_{s, r}^{k, n+1}=E_{s b, r b}^{k-p, n+1}+E_{s a, r a}^{k-q, n+1}$. Now one of $Z E_{s b, r b}^{k-p, n+1}$, $Z E_{s a, r a}^{k-q, n+1}$ is zero and the other is an element of $S$. Thus $1_{F_{l}} V^{k, n} 1_{F_{l}} \in$ $C^{*}(S)=F_{l}$.

Lemma 3.4 Consider the diagram describing a set of generators $S(n, L, K)$ and the $C^{*}$-algebra $C^{*}(S(n, L, K))$. Suppose $l_{1}, \ldots, l_{m}$ are the numbers of those dots in the lower row of the diagram that are not related by mers to dots further left. Then by lemma 3.3 there is a full matrix algebra $F_{l_{i}}$, a direct summand of $C^{*}(S(n, L, K))$ containing the matrix units of the $l_{i}$-th dot. We have in fact $C^{*}(S(n, L, K)) \cong F_{l_{1}} \oplus \ldots \oplus F_{l_{m}}$. Furthermore, the multiplicity with which a dot is contained in $F_{l_{i}}$ is given by the number of 
ways in which it is related by polymers to dot $l_{i}$. Finally, the inclusion of the sub-C $C^{*}$-algebra generated by just the dots into $C^{*}(S(n, L, K))$ is unital.

Thus if we look back at diagram 3 (even though this is not of the form $\left.C^{*}(S(n, L, K))\right)$ we see that the algebra generated by the elements shown has three direct summands, of size $3 \cdot 2^{(n+1)}, 5 \cdot 2^{(n+1)}$ and $3 \cdot 2^{(n+1)}$. Dots $k-2 q, k-p-q$ and $k-2 p$ include into these, one dot into each summand in the order shown, each with multiplicity one. Also dot $k-q$ includes with multiplicities one, one and zero respectively into these summands, $\operatorname{dot} k-p$ with multiplicities zero, one and one, and dot $k$ with multiplicities one, two and one.

Proof: It is convenient at this point to generalise our notation from lemma 3.3. We add a superscript to the symbols $S$ and $A_{m}$ defined there to indicate the dot in question, so the sets in lemma 3.3 will now be denoted $S^{l}$ and $A_{m}^{l}$. The final comment about the inclusion being unital is obvious.

We show first that the various $F_{l_{i}}$ are orthogonal. Suppose dots $l_{1}$ and $l_{2}$ are not related by polymers to dots further left and $l_{1}<l_{2}$. From our assumption if $v \in S^{l_{1}}$ and $u \in S^{l_{2}}$ we have that $v u=u v=0$, since any element of $S^{l}$ begins with a matrix unit of $\operatorname{dot} \#\left(l_{1}, n+1\right)$ and ends with a matrix unit of a dot related by polymers to $\operatorname{dot} \#\left(l_{1}, n+1\right)$, and similarly for $\operatorname{dot} \#\left(l_{2}, n+1\right)$. We also have that $v^{*} u=u^{*} v=0$ for any $v \in S^{l_{1}}, u \in S^{l_{2}}$, so it remains only to show that $u v^{*}=v u^{*}=0$. We need only consider a $u \in S^{l_{2}}$ and a $v \in S^{l_{1}}$ that end in matrix units for the same dot, in fact the same matrix unit, so suppose $u \in A_{\mu}^{l_{2}}$ and $v \in A_{\nu}^{l_{1}}$, where $l_{2}+|\mu|=l_{1}+|\nu|$. Then since $l_{1}$ and $l_{2}$ are assumed to be unrelated $\mu$ is not a tail of $\nu$, so reading from right to left we must come to a point where $\mu$ and $\nu$ have different letters, $\mu=r q t$, $\nu=x p t$ say, for some words $r, t, x$. Looking at the partial isometries $u$ and 
$v$ as words in the generators we see that there are partial isometries $U_{r}, U_{x}$ and $Y$ such that $U_{r} \in A_{r}^{l_{2}}, U_{r}$ ends in a matrix unit $E_{a s, s a}^{l_{2}+|r|, n+1}, U_{x} \in A_{x}^{l_{1}}$, $U_{x}$ ends the matrix unit $E_{a s, s b}^{l_{1}+|x|, n+1}, u=U_{r} Y$ and $v=U_{x} Y$. That $u v^{*}=0$ now follows from observing that $U_{r}$ and $U_{x}$ have orthogonal supports.

To show that $C^{*}(S(n, L, K)) \cong F_{l_{1}} \oplus \ldots \oplus F_{l_{m}}$ it will suffice to show that the unit of $C^{*}(S(n, L, K))$ is in $F_{l_{1}}+\ldots+F_{l_{m}}$. We shall show that if dot $\#(k, n+1)$ is the fat foot of a mer, then $C^{*}\left(S^{k}\right) \subseteq C^{*}\left(S^{k-p}\right)+C^{*}\left(S^{k-q}\right)$. Since the unit of $\operatorname{dot} \#(k, n+1)$ is in $C^{*}\left(S^{k}\right)$, this will allow us to conclude, by moving right to left through the diagram, that the unit of $C^{*}(S(n, L, K))$ is contained in the sum of the $C^{*}\left(S^{l}\right)$ for dots $\#(l, n+1)$ that are not at the fat feet of mers. These however are exactly the $F_{l_{i}}$ s. So consider a dot $\#(k, n+1)$ that is the fat foot of a mer, and let $v \in S^{k}$. Then $v=E_{w, s a}^{k, n+1} Z$ or $v=E_{w, s b}^{k, n+1} X$ for some $Z$ or $X$ in $C^{*}(S(n, L, K)$ ) (assume the former, a similar argument works for the other case). Observe that

$$
\begin{aligned}
E_{w, w}^{k, n+1}=( & \left.E_{w, a s}^{k, n+1} V^{k, n} E_{s a, w}^{k-q, n+1}\right)\left(E_{w, s a}^{k-q, n+1} V^{k, n *} E_{a s, w}^{k, n+1}\right) \\
& +\left(E_{w, a s}^{k, n+1} V^{k, n} E_{s b, w}^{k-p, n+1}\right)\left(E_{w, s b}^{k-p, n+1} V^{k, n *} E_{a s, w}^{k, n+1}\right)
\end{aligned}
$$

so we have

$$
\begin{aligned}
v=( & \left.E_{w, a s}^{k, n+1} V^{k, n} E_{s a, w}^{k-q, n+1}\right)\left(E_{w, s a}^{k-q, n+1} V^{k, n *} E_{a s, s a}^{k, n+1} Z\right) \\
& +\left(E_{w, a s}^{k, n+1} V^{k, n} E_{s b, w}^{k-p, n+1}\right)\left(E_{w, s b}^{k-p, n+1} V^{k, n *} E_{a s, s a}^{k, n+1} Z\right) .
\end{aligned}
$$

The first expression on the right above is in $C^{*}\left(S^{k-q}\right)$ and the second is in $C^{*}\left(S^{k-p}\right)$

Now finally we determine the multiplicities of the inclusions. Suppose as above we have a set of generators $S(n, L, K)$ and that the corresponding diagram contains dots $\#(l, n+1)$ and $\#(k, n+1)$ where the former is not related by polymers to any dots further left. Consider a minimal projection 
$E_{w, w}^{k, n+1}$ in dot \# $(k, n+1)$. The multiplicity of the inclusion of this dot into $F_{l}$ is the rank of the projection $1_{F_{l}}\left(E_{w, w}^{k, n+1}\right) 1_{F_{l}}$ in $F_{l}$. If $l=k$, then $A_{0}^{l}$ is a set of generators for $\operatorname{dot} \#(k, n+1)$, while $v^{*} v$ is orthogonal to $\operatorname{dot} \#(k, n+1)$ for any $v \in S^{l} \backslash A_{0}^{l}$, so the statement is clear in this case. If $l+|\mu| \neq k$, then $A_{\mu}^{l} \perp E_{w, w}^{k, n+1}$. Thus if there are no words $\mu$ for which $l+|\mu|=k$, in particular if $k<l$, then the multiplicity is zero. Notice that $C^{*}\left(A_{\mu}^{l}\right)$ is a full matrix algebra whose unit, $1_{A_{\mu}^{l}}$, commutes with the matrix units of any dot on the $n+1$ st row, so $1_{F_{l}}\left(E_{w, w}^{k, n+1}\right) 1_{F_{l}}$ is the sum of the projections $1_{A_{\mu}^{l}}\left(E_{w, w}^{k, n+1}\right) 1_{A_{\mu}^{l}}$ where $\mu$ runs over those words such that $l+|\mu|=k$ (we use here the definition of $S(n, L, K))$. Given such a $\mu$, looking at the formula for the elements of $A_{\mu}^{l}$ we see that there is exactly one $v$ in $A_{\mu}^{l}$ for which $v^{*} v\left(E_{w, w}^{k, n+1}\right) v^{*} v=v^{*} v$, while for all other $u \in A_{\mu}^{l}$ we have $u^{*} u E_{w, w}^{k, n+1}=0$. Thus the rank of $1_{F_{l}}\left(E_{w, w}^{k, n+1}\right) 1_{F_{l}}$ in $F_{l}$ is the number of words $\mu$ for which $l+|\mu|=k$. This is exactly the number of ways by which $\operatorname{dot} \#(k, n+1)$ is related by polymers to $\operatorname{dot} \#(l, n+1)$.

Proof of theorem 3.2: It follows easily from lemmas 3.3, 3.4 and the discussion preceding lemma 3.3 that $A(p, q)$ is AF. To show that $A(p, q)$ is simple we use the following simple observation: If $p$ and $q$ are coprime natural numbers, then for some natural number $N(p, q)$ all numbers greater than $N(p, q)$ may be written as sums of positive multiples of $p$ and $q$. Notice that in the diagram for $S(n, L, K)$ the direct summands of $C^{*}(S(n, L, K))$ are labeled by the last $q$ dots to the left on the lower row, as these dots are unrelated by mers, and every other dot is related to at least one of these by a polymer. Consider the following increasing sequence of sub- $C^{*}$-algebras of $A(p, q)$.

$$
A_{n}=A\left(S\left(n,-K_{n}, K_{n}\right)\right)
$$


where $K_{1}=1$ and $K_{n+1}=K_{n}+N(p, q)+1+q$. Repeated applications of relations (4) and (7) show that $A(p, q)$ is the inductive limit of the sequence $A_{1} \subseteq A_{2} \subseteq \ldots$ Relations (4) and (7) define a map from $C^{*}\left(S\left(n,-K_{n}, K_{n}\right)\right)$ to $C^{*}\left(S\left(n+1,-K_{n+1}, K_{n+1}\right)\right)$ by sending the terms on the left, read in the first $C^{*}$-algebra to the terms on the right, read in the second $C^{*}$-algebra. Call the limit of this sequence $C$, and write $C_{n}$ for $C^{*}\left(S\left(n,-K_{n}, K_{n}\right)\right)$. The analysis carried out above implies that the map $C_{n} \rightarrow C_{n+1}$ sends each minimal direct summand of $C_{n}$ with non-zero multiplicities into every minimal direct summand of $C_{n+1}$. It follows from this that all of these maps are injective and that $C$ is a simple $C^{*}$-algebra. We get a commutative diagram:

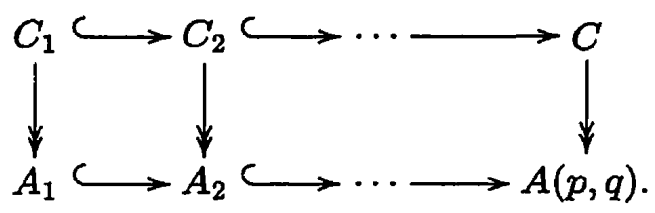

Since $C$ is simple the last downward map is an isomorphism, (with inverse given by the universal property of $A(p, q)$ ), and hence so are all the downward maps at the finite stages. In fact it is now easy to see that $A(S(n, L, K)) \cong$ $C^{*}(S(n, L, K))$ by the obvious map for any $S(n, L, K)$, and we henceforth identify them in this way.

It is easy to see that the map $\alpha$ is a bijection of the set of generators with itself that preserves the relations and so extends to an automorphism $\hat{\alpha}$ of $A(p, q)$.

The proof is concluded by showing that there exists a covariant homomorphism of $(A(p, q), \hat{\alpha})$ onto $\left(\mathrm{O}_{2} \rtimes_{\alpha^{\lambda}} \mathrm{T}, \hat{\alpha}^{\lambda}\right)$ and referring to [Bl] or [OP] (that such a map must be an isomorphism follows from simplicity of $A(p, q))$. We have already observed that the $E$ 's along with relations 1-4 give a presenta- 
tion of $e\left(\left(M_{2 \infty} \otimes K\right) \rtimes_{\tilde{\alpha}} \mathbb{T}\right) e$. The explicit correspondence is given as follows. If we identify the letter a with 1 and b with 2 , then the matrix units $E_{w, w}$ for $w, v \in W^{n}$ are in a natural correspondence with the matrix units of $M_{2^{n}}$ coming from its expression as an n-fold tensor product of copies of $M_{2}$. With this identification $E_{v, w}^{k, n}$ gets mapped to the corresponding matrix unit in the $k$-th direct summand of $M_{2^{n}} \otimes C_{0}(\mathbb{Z})$ in our direct limit decomposition of $e\left(\left(M_{2} \infty K\right) \rtimes_{\bar{\alpha}} \mathbb{T}\right) e$. Let $V$ denote the adjoined unitary in the multiplier algebra of $\left(M_{2^{\infty}} \otimes K\right) \rtimes_{\bar{\alpha}} \mathbb{T} \rtimes_{\bar{\beta}} \mathbb{Z}$ implimenting the action $\tilde{\beta}^{\prime \prime}$. Then if we assume the identification of the matrix units with the $E$ 's already made, we may send $V^{k, n}$ to $\sum_{w \in W^{n}}\left(E_{w, w}^{k, n} V e\right)$ and check that relations 1-7 are satisfied. Straightforward computations then show that the *-homomorphism into $\mathrm{O}_{2} \rtimes_{\alpha^{\lambda}} \mathrm{T}$ given by the universal property of $A(p, q)$ has a dense image and carries $\hat{\alpha}$ onto $\hat{\alpha}^{\prime \prime}$.

Definition 3.5 In [ELP] the following definitions are given. A zero dimensional $N C C W$-complex is just a finite dimensional $C^{*}$-algebra. A one dimensional NCCW-complex is a pullback of the following form. Let $F_{1}$ and $F_{2}$ be two finite dimensional $C^{*}$-algebras with unital maps $\alpha_{1}, \alpha_{2}: F_{1} \rightarrow F_{2}$. Let $e v(0), e v(1)$ denote the maps from $F_{2} \otimes C[0,1]$ to $F_{2}$ given by evaluation at zero and one respectively. We then form the pullback of the following diagram:

$$
F_{2} \otimes C[0,1] \underset{e v(0) \oplus e v(1)}{\left.\alpha_{1} \oplus \alpha_{2}\right|^{F_{1}}} F_{2} \oplus F_{2} .
$$

We shall weaken these requirements slightly. Define a non-unital one dimensional NCCW-complex as a pull-back as above, but without the requirement that the maps $\alpha_{1}$ and $\alpha_{2}$ be unital. Given a description of a non-unital one di- 
mensional NCCW-complex as above we call $F_{1}$ the zero skeleton, $F_{2} \otimes C[0,1]$ the one-cell and the map $\alpha_{1} \oplus \alpha_{2}$ the attaching map.

We then have the following corollary.

Corollary 3.6 For any rational number $\lambda \in \mathbb{Q} \cap(0, \infty) \backslash\{1\}, O_{2} \rtimes_{\alpha^{\lambda}} \mathbb{R}$ is an inductive limit of non-unital one dimensional NCCW-complexes with the property that every direct summand of the zero skeleton is included with non-zero multiplicity into each direct summand of the fibre of the one-cell at both endpoints by the attaching map.

Proof: Observe that both $A_{n}$ and $\hat{\alpha}\left(A_{n}\right)$ are included in $A_{n+1}$ in the fashion described. If we let $B_{n}$ denote the non-unital NCCW-complex consisting of continuous functions from $[0,1]$ into $A_{n+1}$ that are in $A_{n}$ at zero and whose value at one is $\hat{\alpha}$ of their value at zero, we get an increasing sequence $B_{1} \subseteq B_{2} \subseteq, \ldots$ of sub- $C^{*}$-algebras of $M_{\hat{\alpha}}(A(p, q))$ whose union is dense. 


\section{§4. Stable Relations and the Main Result for $\mathrm{O}_{2}$}

In this section we shall require some results about stable relations. We refer the reader to [L] and [ELP] for details.

We use the notation $C^{*}\langle G \mid R\rangle$ to denote the universal $C^{*}$-algebra with generators $G$ subject to the relations $R$, if it exists. $C^{*}\left\langle\left\{x_{1}, \ldots, x_{n}\right\} \mid\left\|x_{i}\right\| \leq 2\right\rangle$ does exist and we shall denote it by $F_{n}^{(2)}$. In the following we shall regard relations among $n$ variables to be just elements of this $C^{*}$-algebra. We say that a $C^{*}$-algebra $C^{*}\langle G \mid R\rangle$ is finitely presented if $R$ and $G$ are both finite sets. As observed in [ELP 2.2.5] this is no real constraint on $R$.

Definition 4.1 (cf. [L 13.2.2]) The $n$-tuple $\left(a_{1}, \ldots, a_{n}\right)$ of elements in a $C^{*}$-algebra $A$ is called a representation of a set of relations $R \subseteq F_{n}^{(2)}$ if $\left\|a_{j}\right\| \leq 2$ and $\Phi_{\mathbf{a}}(p)=0$ for all $p \in R$, where $\Phi_{a}: F_{n}^{(2)} \rightarrow A$ is induced by $x_{i} \mapsto a_{i}$. If for some $\delta<1$ only $\left\|\Phi_{\mathbf{a}}(p)\right\| \leq \delta$ is required to hold for all $p \in R$, then $\left(a_{1}, \ldots, a_{n}\right)$ is called a $\delta$-representation.

Definition 4.2 (cf. [L 13.2.1]) By a finite, bounded set of relations in $n$ variables we mean a finite subset $R$ of $F_{n}^{(2)}$ such that $\left\|x_{j}+I_{R}\right\| \leq 1$, where $I_{R}$ is the ideal generated by $R$.

Definition 4.3 A finite, bounded set of relations $R$ in $F_{n}^{(2)}$ is said to be weakly stable if for every $\varepsilon>0$ there exists a $\delta>0$ such that if $\left(a_{1}, \ldots, a_{n}\right)$ is a $\delta$-representation of $R$ in a $C^{*}$-algebra $A$ there exists a representation $\left(b_{1}, \ldots, b_{n}\right)$ of $R$ in $A$ such that $\left\|a_{i}-b_{i}\right\|<\varepsilon$ for $i=1, \ldots, n$.

It follows from the results of [ELP] and [L] that any non-unital one dimensional NCCW-complex can be finitely presented with a weakly stable set of relations (in fact we may assume just one relation).

Lemma 4.4 A projectionless quotient of a non-unital one dimensional 
NCCW-complex having the property that every direct summand of the zero skeleton is mapped with non-zero multiplicity into every direct summand of the fibre of the one-cell at both endpoints by the attaching map is a non-unital one dimensional NCCW-complex.

Proof: Suppose we are given a non-unital one dimensional NCCW-complex satisfying our hypotheses: $A$ is isomorphic to the pullback of $F_{1}$ and $F_{2} \otimes$ $C[0,1]$ with $\alpha_{1} \oplus \alpha_{2}: F_{1} \rightarrow F_{2} \oplus F_{2}$ and $e v(0) \oplus e v(1): F_{2} \otimes C[0,1] \rightarrow F_{2} \oplus F_{2}$ where $F_{1}$ and $F_{2}$ are finite dimensional algebras and both $\alpha_{1}$ and $\alpha_{2}$ map every direct summand of $F_{1}$ into every direct summand of $F_{2}$ with non-zero multiplicity. Then the primitive spectrum of $A$, denoted $\sigma(A)$, is the union of a finite set, identified with the spectrum of $F_{1}$, and a finite number of copies of $\mathbb{R}$, identified with $\sigma\left(F_{2} \otimes C_{0}(0,1)\right)$, with the topology specified as follows (cf. [E3]). The relative topology on $\sigma\left(F_{1}\right)$ is the discrete topology, each line in $\sigma\left(F_{2} \otimes C_{0}(0,1)\right)$ is open in $\sigma(A)$ and homeomorphic to a line in the relative topology, taking the union of any line in $\sigma\left(F_{2} \otimes C_{0}(0,1)\right)$ with any point of $\sigma\left(F_{1}\right)$ with the relative topology gives the one point compactification of the line, and every point in $\sigma(A)$ is closed.

Let $I$ be a proper ideal in $A$. The spectrum of $I$ is then identified in a natural way with an open subset of $\sigma(A)$, and the spectrum of $A / I$ is homeomorphic to the compliment $\sigma(A) \backslash \sigma(I)$. We shall first see what the set $\sigma(I)$ can be.

Consider $\sigma(I) \cap \sigma\left(F_{1}\right)$. Suppose this set is not empty and is not all of $\sigma\left(F_{1}\right)$. Since it is not empty, there is some neighbourhood of a point in $\sigma\left(F_{1}\right)$ contained in it, and this neighbourhood must contain the ends of all of the lines. Thus any point in $\sigma\left(F_{1}\right) \backslash \sigma(I)$ is an isolated point of $\sigma(A / I)$, which therefore has an elementary $C^{*}$-algebra as a direct summand, and in particular has projections. 
Suppose that $\sigma(I) \supseteq \sigma\left(F_{1}\right)$. Then we have that $\sigma(A / I)$ is contained in the union of finitely many closed intervals, and $A / I$ is a quotient of a finite direct sum of matrix algebras over $C[0,1]$. As these are unital, so is $A / I$, in particular, it has a projection.

We have reduced the proof of our lemma to the case in which $\sigma(I)$ is contained in $\sigma\left(F_{2} \otimes C(0,1)\right)$. If we write $\sigma\left(F_{2} \otimes C(0,1)\right) \cong L_{1} \amalg \ldots \amalg L_{k}$ where each $L$ is homeomorphic to $\mathbb{R}$, it is easy to see that, if $A / I$ is projectionless, then for each $i L_{i} \cap \sigma(I)$ must be connected (otherwise $A / I$ would contain a quotient of a matrix algebra over $C[0,1])$. Furthermore, $\sigma(I)$ can not intersect all of the $L_{i}$ 's non-trivially or we would again have projections in the quotient. Thus we have only to consider the case in which $\sigma(I)$ is a disjoint union of open intervals, at most one in each $L_{i}$, and not in every $L_{i}$. We show that in this case the quotient is a non-unital one dimensional NCCW-complex. Suppose $F_{2}=A_{1} \oplus \ldots \oplus A_{k}$, where each $A$ is a full matrix algebra, and let $\varphi_{j}^{(1)}, \varphi_{j}^{(2)}$ denote the maps from $F_{1}$ to the $j$-th minimal direct summand of $F_{2}$ at the left and right endpoints respectively. Suppose further that the ideal $I$ cuts the lines $L_{l}$ to $L_{k}$. Define $F_{1}^{\prime}$ and $F_{2}^{\prime}$ as follows. $F_{1}^{\prime}=F_{1} \oplus\left(A_{l}^{1} \oplus \ldots \oplus A_{k}^{1}\right) \oplus$ $\left(A_{l}^{2} \oplus \ldots \oplus A_{k}^{2}\right)$ and $F_{2}^{\prime}=\left(A_{1} \oplus \ldots \oplus A_{l-1}\right) \oplus\left(A_{l}^{1} \oplus \ldots \oplus A_{k}^{1}\right) \oplus\left(A_{l}^{2} \oplus \ldots \oplus A_{k}^{2}\right)$, where the superscripts are just to distinguish the two copies. Define maps $\alpha_{1}^{\prime}, \alpha_{2}^{\prime}$ from $F_{1}^{\prime}$ to $F_{2}^{\prime}$ as follows. $\alpha_{1}^{\prime}$ maps $F_{1}$ into the 1-st $l-1$ summands of $F_{2}^{\prime}$ by $\varphi_{1}^{(1)}, \ldots, \varphi_{l-1}^{(1)}$ respectively. It maps $F_{1}$ into $A_{l}^{1}, \ldots, A_{k}^{1}$ by $\varphi_{l}^{(1)}, \ldots, \varphi_{k}^{(1)}$ respectively. $\alpha_{1}^{\prime}$ maps $A_{l}^{1}, \ldots, A_{k}^{1}$ to $A_{l}^{2}, \ldots, A_{k}^{2}$ respectively each with the identity map. All the other partial maps for $\alpha_{1}^{\prime}$ are zero. $\alpha_{2}^{\prime}$ maps $F_{1}$ into the first $l-1$ summands of $F_{2}^{\prime}$ by $\varphi_{1}^{(2)}, \ldots, \varphi_{l-1}^{(2)}$ respectively. It maps $F_{1}$ into $A_{l}^{2}, \ldots, A_{k}^{2}$ by $\varphi_{l}^{(2)}, \ldots, \varphi_{k}^{(2)}$ respectively, it maps $A_{l}^{2}, \ldots, A_{k}^{2}$ to $A_{l}^{1}, \ldots, A_{k}^{1}$ respectively, each with the identity map, and all of the other partial maps for $\alpha_{2}^{\prime}$ are zero. It is then easy to see that $A / I$ is isomorphic to the non-unital 
one dimensional NCCW-complex with zero-skeleton $F_{1}^{\prime}$, one-cell $F_{2}^{\prime} \otimes C[0,1]$ and attaching map $\alpha_{1}^{\prime} \oplus \alpha_{2}^{\prime}$.

We shall refer to the additional condition on a non-unital one dimensional NCCW-complex mentioned in lemma 4.4 as the endpoint property.

Definition 4.5 We shall say that a separable $C^{*}$-algebra $A$ has the local approximation property with respect to a class of $C^{*}$-algebras $\mathfrak{C}$ if for every finite set $F$ of elements of $A$ and every $\varepsilon>0$ there is a $C \in \mathcal{C}$ and a *homomorphism $\varphi: C \rightarrow A$ such that each element of $F$ lies within $\varepsilon$ of the image of $\varphi$.

Lemma 4.6 If a separable projectionless $C^{*}$-algebra has the local approximation property with respect to the class of non-unital one dimensional NCCW-complexes having the endpoint property, then it is an inductive limit of a sequence of non-unital one dimensional NCCW-complexes.

Proof: From lemma 4.4 we may assume that the algebra has the local approximation property with injective maps for the class of non-unital one dimensional NCCW-complexes. The statement now follows from [L 15.2.2].

We shall now need some results about continuous fields of $C^{*}$-algebras. We refer the reader to [Dix] for a more thorough discussion.

Definition 4.7 A continuous field of $C^{*}$-algebras consists of three things: A topological space $X$, for each point $x \in X$ a $C^{*}$-algebra $A_{x}$, and a set of sections $\Gamma \subseteq \prod_{x \in X} A_{x}$ that satisfies the following axioms:

1. Endowed with the pointwise operations, $\Gamma$ forms a *-algebra;

2. For every point $x \in X$, the set $\{f(x) \mid f \in \Gamma\}$ is dense in $A_{x}$;

3. The functions $x \mapsto\|f(x)\|$ for $f \in \Gamma$ are continuous on $X$;

4. If $g \in \prod_{x \in X} A_{x}$ and for every point $t \in X$ and $\varepsilon>0$ there is a 
neighbourhood $V$ of $t$ and an $f \in \Gamma$ such that $\|g-f\|<\varepsilon$ on $V$, then $g \in \Gamma$.

A set of sections $\Gamma$ that only satisfies axioms 1-3 is called a continuity structure on $\prod_{x \in X} A_{x}$. If one has a continuity structure $\Gamma$, then one may enlarge $\Gamma$ to a set of sections satisfying all four axioms.

Example 4.8 The simplest example of a continuous field of $C^{*}$-algebras is given by a tensor product, $A \otimes C_{0}(X)$, where $X$ is a locally compact Hausdorff space. In this case $A_{x} \cong A$ for all $x \in X$ and $\Gamma$ is the set of continuous $A$ valued functions on $X$ vanishing at infinity.

Lemma 4.9 Let $\delta$ and $\varepsilon$ such that $1>\delta>0$ and $1-\delta>\varepsilon>0$ be given. Let $\{A(t) \mid t \in X\}$ be a continuous field of $C^{*}$-algebras over a topological space $X$ with continuous sections $\Gamma, t_{0} \in X$, and suppose that $y_{1}, \ldots, y_{n} \in \Gamma$ are such that $\left\|y_{i}\left(t_{0}\right)\right\|<2$ and $\left(y_{1}\left(t_{0}\right), \ldots, y_{n}\left(t_{0}\right)\right)$ is a $\delta$-representation of a relation $r \in F_{n}^{(2)}$. Then there is a neighbourhood $V$ of $t_{0}$ such that for all $s \in V,\left(y_{1}(s), \ldots, y_{n}(s)\right)$ is a $(\delta+\varepsilon)$ - representation of $r$.

Proof: Let $P$ be a *-polynomial in the generators $x_{1}, \ldots, x_{n}$ of $F_{n}^{(2)}$ such that $\|P-r\|<\varepsilon / 3$ in $F_{n}^{(2)}$. Since $s \mapsto\left\|P\left(y_{1}(s), \ldots, y_{n}(s)\right)\right\|$ is continuous on $X$ there is some neighbourhood $V$ of $t_{0}$ on which it varies by less than $\varepsilon / 3$ and on which $\left\|y_{i}(\cdot)\right\|<2$. If $\psi_{s}: F_{n}^{(2)} \rightarrow A(s)$ denotes the map induced by $x_{i} \mapsto y_{i}(s)$, which is well defined for $s \in V$, then $\left\|\psi_{s}(r)\right\|<\delta+\varepsilon$ for all $s$ in $V$.

Lemma 4.10 Let $\{A(t) \mid t \in X\}$ be a continuous field of $C^{*}$-algebras over a topological space $X$, and suppose that $B \cong C^{*}\left\langle x_{1}, \ldots, x_{n} \mid r\right\rangle$ is a finitely generated $C^{*}$-algebra described by a weakly stable relation $r$. Let $\varepsilon>0$, $t_{0} \in X$ and continuous sections $y_{1}, \ldots y_{m}$ be given. Suppose that there is a ${ }^{*}$ homomorphism $\psi_{t_{0}}: B \rightarrow A\left(t_{0}\right)$ such that $\left\{y_{1}\left(t_{0}\right), \ldots, y_{m}\left(t_{0}\right)\right\} \subseteq \varepsilon / 2 \psi_{t_{0}}(B)$. 
Then there is a neighbourhood $V$ of $t_{0}$ such that for every $s \in V$ there is a *-homomorphism $\psi_{s}: B \rightarrow A(s)$ with $\left\{y_{1}(s), \ldots, y_{m}(s)\right\} \subseteq_{\varepsilon} \psi_{s}(B)$.

Proof: Find *-polynomials $p_{1}, \ldots, p_{m}$ in $n$ variables such that $\left\|p_{j}\left(\psi_{t_{0}}\left(x_{1}\right), \ldots, \psi_{t_{0}}\left(x_{n}\right)\right)-y_{j}\left(t_{0}\right)\right\|<3 \varepsilon / 4$ for $j=1, \ldots, m$. Find continuous sections $z_{1}, \ldots, z_{n}$ such that $z_{i}\left(t_{0}\right)=\psi_{t_{0}}\left(x_{i}\right)$ for $i=1, \ldots, n$. There exists a neighbourhood $U$ of $t_{0}$ such that for all $s \in U$ and for each $j$, $\left\|p_{j}\left(z_{1}(s), \ldots, z_{n}(s)\right)-y_{j}(s)\right\|<3 \varepsilon / 4$. Choose $\varepsilon^{\prime}$ such that if $a_{1}, \ldots, a_{n}$, $a_{1}^{\prime}, \ldots, a_{n}^{\prime}$ are elements in a $C^{*}$-algebra and $\left\|a_{i}-a_{i}^{\prime}\right\|<\varepsilon^{\prime}$ for $i=1, \ldots, n$, then for each $j,\left\|p_{j}\left(a_{1}, \ldots, a_{n}\right)-p_{j}\left(a_{1}^{\prime}, \ldots, a_{n}^{\prime}\right)\right\|<\varepsilon / 4$. There exists a $\delta$ such that if $\left(w_{1}, \ldots w_{n}\right)$ is a $\delta$-representation of $r$ in a $C^{*}$-algebra, then there exists a representation $\left(w_{1}^{\prime}, \ldots, w_{n}^{\prime}\right)$ of $r$ with $\left\|w_{i}-w_{i}^{\prime}\right\|<\varepsilon^{\prime}$ for each $i$. By lemma 4.9 above there exists a neighbourhood $W$ of $t_{0}$ such that if $s \in W$ then $\left(z_{1}(s), \ldots, z_{n}(s)\right)$ is a $\delta$-representation of $r$. Thus for $s \in W$ there exists a representation $\left(z_{1}^{s}, \ldots z_{n}^{s}\right)$ of $r$ in $A(s)$ with $\left\|z_{i}^{s}-z_{i}(s)\right\|<\varepsilon^{\prime}$ for each $i$. Define $\psi_{s}: B \rightarrow A(s)$ for each $s \in U \cap W$ by $x_{i} \mapsto z_{i}^{s}$ for $i=1, \ldots, n$. Now it is easy to see that for $s \in U \cap W$ we have $\left\{y_{1}(s), \ldots, y_{m}(s)\right\} \subseteq_{\varepsilon} \psi_{s}(B)$.

It follows from 3.6 of [Rie] that the $\mathrm{O}_{2} \rtimes_{\alpha^{\lambda}} \mathbb{R}$ s fit into a continuous field over $\mathbb{R}$ with the image of any fixed element of $C_{c}\left(\mathbb{R}, O_{2}\right)$ in each crossed product giving a continuous section. (cf. also [ENN].)

Corollary 4.11 Suppose $t \in \mathbb{Q} \cap(0, \infty) \backslash\{1\}, \varepsilon>0$, and $f_{1}, \ldots f_{n} \in$ $C_{c}\left(\mathbb{R}, \mathrm{O}_{2}\right)$ are given. Then there exists a neighbourhood $U$ of $t$, a non-unital one dimensional NCCW-complex $A$ with the endpoint property, and, for every $s \in U, a{ }^{*}$-homomorphism $\psi_{s}: A \rightarrow \mathrm{O}_{2} \rtimes_{\alpha^{s}} \mathbb{R}$ such that $\left\{\varphi_{s}\left(f_{1}\right), \ldots, \varphi_{s}\left(f_{n}\right)\right\} \subseteq_{\varepsilon} \psi_{s}(A)$, where $\varphi_{s}$ denotes the canonical inclusion of $\mathrm{C}_{c}\left(\mathbb{R}, \mathrm{O}_{2}\right)$ into $\mathrm{O}_{2} \rtimes_{\alpha} \mathbb{R}$.

Proof: The corollary is immediate from lemma 4.10 above and corollary 
3.6.

Theorem 4.12 (The main result for $\mathrm{O}_{2}$ ) The set of irrational numbers $\lambda$ for which $\mathrm{O}_{2} \rtimes_{\alpha^{\lambda}} \mathbb{R}$ is an inductive limit of non-unital one dimensional NCCW-complexes is a dense set of Baire category 2 in $(0, \infty)$.

Proof: From lemma 4.6 we only have to show that the local approximation property with respect to the class of non-unital one dimensional NCCWcomplexes with the endpoint property holds for such a set. Pick a countable dense subset of $C_{c}\left(\mathbb{R}, \mathrm{O}_{2}\right)$ and call it $\mathfrak{G}$. To conclude that a given $\mathrm{O}_{2} \rtimes_{\alpha^{\alpha}} \mathbb{R}$ has the local approximation property it will suffice to show that the elements in $\varphi_{s}(\mathfrak{G})$ may be approximated. From corollary 4.11 above, for each finite subset $F \subseteq \mathfrak{G}, \varepsilon>0$, and each $t \in \mathbb{Q} \cap(0, \infty) \backslash\{1\}$ there is a neighbourhood $V(t, F, \varepsilon)$ of $t$, a non-unital one dimensional NCCW-complex $B(t, F \varepsilon)$ with the endpoint property and for every $s \in V(t, F, \varepsilon)$ a *-homomorphism $\psi(t, s, F, \varepsilon): B(t, F, \varepsilon) \rightarrow O_{2} \rtimes_{\alpha^{s}} \mathbb{R}$ such that $\varphi_{s}(F) \subseteq_{\varepsilon} \psi(t, s, F, \varepsilon) B(t, F, \varepsilon)$. Let $G(\varepsilon, F)=\cup_{t \in \mathbb{Q}}(0, \infty) \backslash\{1\} V(t, F, \varepsilon)$. Then for every $s \in G(\varepsilon, F), \varphi_{s}(F)$ is approximately contained to within $\varepsilon$ by the image of a non-unital one dimensional NCCW-complex with the end point property. Observe that $G(\varepsilon, F)$ contains a dense open set. Let $\varepsilon_{n}$ be a sequence of positive numbers converging to zero and let $\mathfrak{F}(\mathfrak{G})$ denote the set of finite subsets of $\mathfrak{G}$. Then the set $G=\cap_{F \in \mathfrak{I}(*)} \cap_{\varepsilon_{n}} G\left(\varepsilon_{n}, F\right)$ is contained in the set of points $s$ in $(0, \infty)$ for which $\mathrm{O}_{2} \rtimes_{\alpha^{s}} \mathbb{R}$ has the local approximation property, and $G$ clearly contains a dense $G_{\delta}$ set. It follows that $G \backslash \mathbb{Q}$ is a dense set of Baire category 2. 


\section{§5. $O_{n}$ and Closing Remarks}

In this section we shall describe how the results of sections 2-4 generalise to $O_{n}$ for $n>2$. As the statements and their proofs closely resemble those for $\mathrm{O}_{2}$, we shall be brief.

The actions of $\mathbb{R}$ on $O_{n}$ that we are considering depend on $n$ real parameters $\lambda_{1}, \ldots, \lambda_{n}$. Omitting the trivial case and rescaling we may assume that $\lambda_{1}=$ 1. By [Rie], we obtain a continuous field of $C^{*}$-algebras over $\mathbb{R}^{n-1}$ with fibres $O_{n} \rtimes_{\alpha^{\lambda}} \mathbb{R}$, where $\lambda=\left(1, \lambda_{2}, \ldots, \lambda_{n}\right)$ is a multi-index. As in the case of $O_{2}$ we shall analyse the fibres for which $\alpha^{\lambda}$ is periodic, that is, when $\lambda_{1}, \ldots, \lambda_{n}$ are all rational numbers. If $\lambda=\left(1, \lambda_{2}, \ldots, \lambda_{n}\right)$ with $\lambda_{2}, \ldots, \lambda_{n} \in \mathbb{Q}$, we may, by rescaling the real parameter, get a new action, also to be called $\alpha^{\lambda}$, with parameters $p_{1}, \ldots, p_{n} \in \mathbb{Z}$ such that $\operatorname{gcd}\left(p_{1}, \ldots, p_{n}\right)=1$. We may restrict attention to the case in which the ps are all distinct. Now [Bl2 prop. 10.3.2] may be applied to conclude that $O_{n} \rtimes_{\alpha^{\lambda}} \mathbb{R} \cong M_{\hat{\alpha}^{\lambda}}\left(O_{n} \rtimes_{\alpha^{\lambda}} \mathbb{T}\right)$. We proceed to analyse $O_{n} \rtimes_{\alpha^{\lambda}} \mathbb{T}$ in the same way as for the special case of $O_{2}$, writing $O_{n}$ as $e\left(M_{n} \infty X \rtimes_{\beta} \mathbb{Z}\right) e$ etc. We may again display the results of this analysis in diagrams.

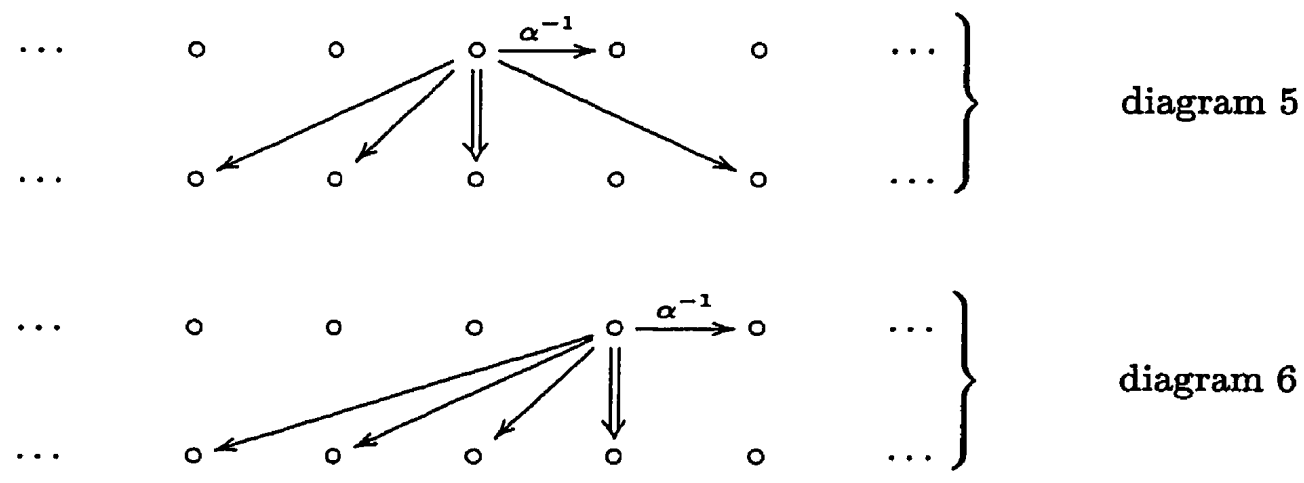

In these diagrams, a dot in the $j$ th row represents a copy of $M_{n^{j}}$ and the dots and thin arrows yield a Bratteli diagram for an inductive limit of algebras 
$M_{n^{j}} \otimes C_{0}(\mathbb{Z})$. The number of thin downward arrows emanating from each $\operatorname{dot}$ is $n$, the fat arrows, as before denote partial isometries setting minimal projections in the upper dots equivalent to minimal projections in the lower dots. The finite case is distinguished here by having all of the thin arrows on the same side of the fat arrow (in other words, all the parameters of the same sign). By an analysis similar to that carried out in section 3 for the special case of $\mathrm{O}_{2}$, one can show that the universal $C^{*}$-algebra described by a diagram such as diagram 6 above is an AF algebra. Using the observation that if $p_{1}, \ldots, p_{n}$ are natural numbers with greatest common divisor 1 , then there some natural number $N$ such that any $k \geq N$ may be written as a sum of positive multiples of $p_{1}, \ldots, p_{n}$; we again obtain an inductive system for this algebra in which each minimal direct summand of the $n$-th algebra is mapped with non-zero multiplicity into each minimal direct summand of the $n+1$-st algebra. From this analogues of theorem 3.2 and corollary 3.6 follow. The arguments of section 4 apply unchanged to prove the following.

Theorem 5.1 (The main result for $O_{n}$ ) The set of points $\left(\lambda_{2}, \ldots, \lambda_{n}\right)$ in $(0, \infty)^{n-1}$ for which $O_{n} \rtimes_{\alpha^{\lambda}} \mathbb{R}$ is an inductive limit of non-unital one dimensional NCCW-complexes contains a dense $G_{\delta}$ set.

Remark 5.2 The diagrammatic representation of the $A(p, q)$ 's allows one to read off fairly simple expressions for their K-theory. In section 3 we found that the minimal direct summands of $C^{*}(S(n, L, K))$ are labelled by the $q$ leftmost dots in the lower row of the diagram for $S(n, L, K),(q>p)$, and that the minimal projections for these dots are minimal in there respective summands of $C^{*}(S(n, L, K))$. Thus the ordered $K_{0}$ group of $A(p, q)$ is a direct limit of copies of the simplicial group $\mathbb{Z}^{q}$.

It is easy to see that for any projection in one of the $C^{*}(S(n, L, K))$ 's, $A(p, q)$ 
contains an equivalent orthogonal projection. Since $A(p, q)$ is a simple AF algebra its $K_{0}$ group is a simple ordered group, so every element is an order unit. We thus conclude that the scale of $K_{0}(A(p, q))$ is the whole positive cone, and that $A(p, q)$ is stable.

It remains only to determine the multiplicity matrices for the maps $\mathbb{Z}^{q} \rightarrow \mathbb{Z}^{q}$ for some suitably chosen nest of finite dimensional sub- $C^{*}$-algebras. We shall describe recursively a sequence of sub-diagrams of the diagram for $A(p, q)$, and the sub- $C^{*}$-algebras will be those given by the generators represented in these sub-diagrams. The first sub-diagram, $D_{0}$, is just $S(0,0,0) . D_{n}$ is a sub-diagram of the diagram for $A(p, q)$ that is contained between the $n$-th and $n+1$-st rows of dots. $D_{n+1}$ will be the sub-diagram contained between the $n+1$-st and $n+2$-nd rows given as follows. In the $n+1$-st row $D_{n+1}$ includes all of the dots included in the bottom row of $D_{n}$, and one more on the left. $D_{n+1}$ contains all of the arrows emanating from these dots. In row $n+2, D_{n+1}$ contains the dots at the feet of any of the arrows, fat or thin, in $D_{n+1}$, and any dot between two such. (Note that in this context it would only make sense to talk of omitting fat arrows from such a sub-diagram, as the thin ones represent relations among the generators, not additional generators.) In diagram 7 the leftmost portion of two successive sub-diagrams for $A(1,2)$ is shown.

diagram 7

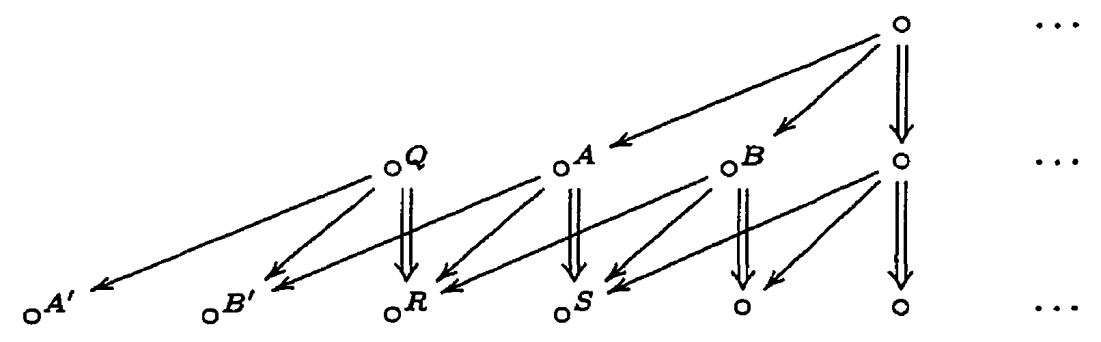

If we denote by $A_{n}$ the sub-C*-algebra of $A(p, q)$ described by $D_{n}$, appli- 
cations of the relations described in section 3 imply that $A_{n} \subseteq A_{n+1}$ and $\overline{\cup A_{n}}=A(p, q)$.

To determine the multiplicity with which a minimal direct summand of $A_{n}$ is included into a minimal direct summand of $A_{n+1}$, we need only check the rank of a minimal projection of the summand of $A_{n}$ when cut down by the unit of the summand of $A_{n+1}$ in question. Even though the $A_{n}$ 's are not strictly speaking of the form $C^{*}(S(n, L, K)$ ), (they differ only in that they may not have an odd number of dots in the top row of their corresponding diagram), the analysis of section 3 may still be applied. A minimal projection in a given minimal direct summand of $A_{n}$ is given by taking a minimal projection in one of the $q$ leftmost dots in the lower row of $D_{n}$, as we saw in section 3. For a particular minimal direct summand of $A_{n+1}$ we find the desired multiplicity by writing one of these projections as a sum of two projections in dots on the $n+2$-nd row and using the rules given in lemma 3.4 for determining the multiplicities with which these dots are included in the minimal direct summands of $A_{n+1}$. Notice that this implies that the matrix describing the multiplicities depends on only the leftmost portion of $D_{n}$ and $D_{n+1}$, as illustrated in diagram 7 , and therefore does not depend on $n$.

We illustrate this procedure for $A(1,2)$. The minimal direct summands for an algebra $A_{n}$ are labelled by the dots marked $A, B$ in the diagram, and those for $A_{n+1}$ by the dots marked $A^{\prime}, B^{\prime}$. To get from $A$ to $A^{\prime}$ by going down a thin arrow and along a polymer there is only one choice: One goes down to $\operatorname{dot} R$, up to $\operatorname{dot} Q$ and down to $A^{\prime}$. To get from $\operatorname{dot} B$ to $\operatorname{dot} A^{\prime}$ one may go down to $R$, up to $Q$ and down to $A^{\prime}$, or down to $S$, up to $A$, down to $R$, up to $Q$ and down to $A^{\prime}$. Thus the summand of $A_{n}$ labelled $A$ includes into 
the summand of $A_{n+1}$ labelled $A^{\prime}$ with multiplicity one, the summand of $A_{n}$ labelled $B$ includes into the same summand with multiplicity two. Doing the other two combinations we conclude that

$$
K_{0}(A(1,2)) \cong \stackrel{\lim }{\longrightarrow}\left\{\mathbb{Z}^{2},\left(\begin{array}{ll}
1 & 2 \\
2 & 3
\end{array}\right)\right\}
$$

As this matrix has determinant $-1, K_{0}(A(1,2)) \cong \mathbb{Z}^{2}$ as a group. Its positive cone is the half space lying above the line through the origin with slope $(1-\sqrt{ } 5) / 2$.

Remark 5.3 Finally, a remark about the diagrams for the infinite case. These are really not important for the object of this paper, but they do perhaps provide some additional insight about the conditions on the projectionless/purely infinite dichotomy found by Kishimoto and Kumjian. Consider diagram 5. Starting at a given dot in this diagram, one may, by going first down to the right several times and then down to the left several times, return by thin arrows to the same column. Thus the fat arrows going straight down set projections in the dot we started with equivalent to sub-projections of themselves, when they are written in terms of elements of a lower row. So in these algebras one can "see" some of the infinite projections. 
[Bl1] B. Blackadar, A simple $C^{*}$-algebra with no non-trivial projections, Proc. Amer. Math. Soc. 78 (1980), 504-508.

[Bl2] B. Blackadar, K-theory for operator algebras, M. S. R. I. Monographs \# 5, Springer Verlag, Berlin and New York, 1986.

[Br] O. Bratteli, Inductive limits of finite dimensional $C^{*}$-algebras, Trans. Amer. Math. Soc. 171 (1972) 195-234.

[Co] A. Connes, An analogue of the Thom isomorphism for Crossed products of a $C^{*}$-algebra by an action of $R$, Advances in Math. 39 (1981) 31-55.

[Cu1] J. Cuntz, Simple $C^{*}$-algebras generated by isometries, Commun. Math. Phys. 57 (1977), 173-185.

[Cu2] J. Cuntz, K-theory for certain $C^{*}$-algebras, Ann. of Math. (2) 113 (1981), 181-197.

[Dix] J. Dixmier, $C^{*}$-algebras, North Holland, Amsterdam, New York, Oxford, 1977.

[EHS] E.G. Effros, D.E. Handelman, and C.L. Shen, Dimension groups and their affine representations, Amer. J. Math. 102 (1980) 385-407.

[ELP] S. Eilers, T. Loring and G. Pedersen, Stability of anti-commutation relations: An application of non-cummutative $C W$-complexes, J. Reine Angew. Math, to appear.

[E1] G.A. Elliott, On the classification of inductive limits of sequences of semi-simple finite-dimensional algebras, J. Algebra 38 (1976) 29-44.

[E2] G.A. Elliott, The classification problem for amenable $C^{*}$-algebras, Proc. ICM, Zurich 1994.

[E3] G.A. Elliott, An invariant for simple $C^{*}$-algebras, Invited Papers/ Articles Sollicités (ed. J.B. Carrell and R. Murty), Canadian Mathematical Society, Ottawa, 1996, 61-90.

[ENN] G.A. Elliott, T. Natsume and R. Nest, The Heisenberg group and K-Theory, K-Theory 7 (1993), 409-428.

[Ev] D.E. Evans, $O_{n} O_{n}$, Publ. RIMS, Kyoto Univ. 16 (1980) 915-927

[K] E. Kirchberg, The classification of purely infinite $C^{*}$-algebras using Kasparov's theory, third draft, preprint, 1994.

[KP] E. Kirchberg and N.C. Phillips, Embedding of exact $C^{*}$-algebras and continuous fields in the Cuntz algebra $\mathrm{O}_{2}$, preprint, 1998.

[Ks] A. Kishimoto, Simple crossed products by locally compact abelian groups, Yokohama Math. J. 28 (1980) 69-85. 
[KK1] A. Kishimoto and A. Kumjian, Simple, stably projectionless $C^{*}$ algebras arising as crossed products, Canadian J. Math. 48 (1996) \# 5 980996.

[KK2] A. Kishimoto and A. Kumjian, Crossed products of Cuntz algebras by quasi-free automorphisms, Fields Institute Communications, Volume 13, (1997), 173-192.

[L] T. Loring, Lifting solutions to perturbing problems in $C^{*}$-algebras, Fields Institute Monograph \# 8, 1997.

[OP] D. Olesen and G. Pedersen, Partially inner $C^{*}$-dynamical systems, J. Functional Analysis 66 (1986), 262-281.

[Ped] G.K. Pedersen, $C^{*}$-algebras and their automorphism groups, Academic Press, London, New York, San Francisco, 1979.

[Rie] M. Rieffel, Continuous fields of $C^{*}$-algebras coming from group cocycles and actions, Mathematische Annalen 283 (1989) 631-643.

[Rør] M. Rørdam, Classification of certain infinite simple $C^{*}$-algebras, J. Functional Analysis 131 (1995) 415-458. 